\title{
Review Article \\ Properties of the Parabolic Anderson Model and the Anderson Polymer Model
}

\author{
Michael Cranston \\ Mathematics Department, University of California, Irvine, CA 92697-3875, USA \\ Correspondence should be addressed to Michael Cranston; mcransto@gmail.com
}

Received 3 September 2012; Accepted 7 November 2012

Academic Editors: M. Campanino and S. Sagitov

Copyright ( 2013 Michael Cranston. This is an open access article distributed under the Creative Commons Attribution License, which permits unrestricted use, distribution, and reproduction in any medium, provided the original work is properly cited.

In this article we examine some properties of the solutions of the parabolic Anderson model. In particular we discuss intermittency of the field of solutions of this random partial differential equation, when it occurs and what the field looks like when intermittency doesn't hold. We also explore the behavior of a polymer model created by a Gibbs measure based on solutions to the parabolic Anderson equation.

\section{Introduction}

It has been twenty years since the publication of the seminal work "Parabolic Anderson Problem and Intermittency" by Carmona and Molchanov. Their memoir has inspired an enormous amount of research on the subject in the intervening years. In this paper we hope to give an account of what is now known about the behavior of solutions of the parabolic Anderson equation as well as the behavior of typical paths under the Anderson polymer measure. Perhaps the initial and still most compelling reason for studying the parabolic Anderson model is physical. In the three-dimensional case it provides a model for the growth of magnetic fields in young stars. In addition, it also has an interpretation as a population growth model. Further, since the work of [1] it has also provided a model for a polymer in a random media. Furthermore, it is a part of the theory of stochastic partial differential equations. Besides its interest as what can be described as a canonical object, in the sense that Brownian motion is a canonical object, its interest also derives from its relations to many other important models. In particular, it is a close relative of other models, for example, the stepping stone model [2], catalytic branching [3], super random walk, the Burger's equation [4], and the KPZ equation [4]. The original motivation of Anderson concerned the question of whether there were bound states for electrons in crystals with impurities. The crystal structure is taken to be $\mathbf{Z}^{d}$ and the impurities are modeled by means of an iid random field $\left\{\xi_{x}\right.$ : $\left.x \in \mathbf{Z}^{d}\right\}$. The phenomena of localization can be expressed as the existence of eigenfunctions of the Hamiltonian $H=\Delta+\xi$.

The equation satisfied by a magnetic field generated by a turbulent flow leads naturally to an analogous parabolic equation with a time varying random field $\{v(t, x): t \in$ $\left.[0, \infty), x \in \mathbf{R}^{3}\right\}$ as opposed to the time stationary field $\left\{\xi_{x}\right.$ : $\left.x \in \mathbf{Z}^{d}\right\}$ which arises in the original localization question. The difference is that the random medium changes rapidly in the case of the magnetic field generated by turbulent flow whereas the impurities in the localization can be considered to be unchanging in time. In the latter case, the fluctuations in the medium are slow compared to the phenomena of interest, capture of electrons.

The magnetic field in a star is generated by the turbulent flow of electrical charges. Turbulent flows are modeled by means of a randomly evolving velocity field, see, for example, [5] or [6] for a canonical example. Let $\{v(t, x): t \in[0, \infty), x \in$ $\mathbf{R}^{3}$ \} denote such a velocity field on $\mathbf{R}^{3}$. Typically, this field is incompressible, Markov in time and Gaussian together with other spectral properties. The magnetic field generated by charges carried by the Lagrangian flow of $\{v(t, x): t \in$ $\left.[0, \infty), x \in \mathbf{R}^{3}\right\}, \mathbf{H}=\left(H_{1}, H_{2}, H_{3}\right)$ is incompressible and evolves according to an equation of the form

$$
\begin{aligned}
\frac{\partial \mathbf{H}_{i}}{\partial t}+\left(v_{j} \nabla_{j}\right) \mathbf{H}_{i} & =\kappa \Delta \mathbf{H}_{i}+\left(\mathbf{H}_{j} \nabla_{j}\right) v_{i}, \\
\mathbf{H}(0, x) & =\mathbf{H}_{0}(x),
\end{aligned}
$$


where in this case $\Delta$ is the standard Laplacian on $\mathbf{R}^{3}$. This equation has been studied in [7-11], to name but a few references. We mention now some of the results of [9], on a tractable version of the model which one gets when $\mathbf{R}^{d}$ is replaced by $\mathbf{Z}^{d}$ and in the environment one takes the time correlation in the velocity field to 0 . In this version of the model, the field $\left\{v(t, x): t \in[0, \infty), x \in \mathbf{R}^{3}\right\}$ is replaced by a $3 \times 3$ matrix Wiener process, $\left\{w(t, x): t \geq 0, x \in \mathbf{Z}^{3}\right\}$ on some probability space $\left(\Omega, \mathscr{F}_{t}, t \geq 0, Q\right)$ satisfying

$$
d w_{i j}(t, x) d w_{k l}(s, y)=\delta_{0}(t-s) \delta_{i k} \delta_{j l} d t .
$$

Then (1) satisfied by $\mathbf{H}$ takes the form

$$
\begin{aligned}
d \mathbf{H}_{i}(t, x)= & \kappa \Delta \mathbf{H}_{i}(t, x) \\
+ & \sum_{j=1}^{3} \mathbf{H}_{j}(t, x) \partial w_{i j}(t, x), \quad i=1,2,3, \\
& \quad \mathbf{H}(0, x)=\mathbf{H}_{0}(x),
\end{aligned}
$$

where $\Delta$, the discrete Laplacian, is given by $\Delta f(x)=$ $\sum_{y:\|x-y\|=1}(f(y)-f(x))$ and $\partial w_{i j}$ is the Stratonovich differential of $w_{i j}$. Converting to the Itô differential instead leads to the following equivalent form:

$$
\begin{aligned}
d \mathbf{H}_{i}(t, x)= & \kappa \Delta \mathbf{H}_{i}(t, x)+\sum_{j=1}^{3} \mathbf{H}_{j}(t, x) d w_{i j}(t, x) \\
+ & \frac{1}{2} \mathbf{H}_{i}(t, x) d t, \quad i=1,2,3, \\
& \mathbf{H}(0, x)=\mathbf{H}_{0}(x) .
\end{aligned}
$$

The average (over the media) of the magnetic field,

$$
\mathbf{M}_{1}(t, x) \equiv E[\mathbf{H}(t, x)],
$$

is easily seen to satisfy

$$
\frac{\partial \mathbf{M}_{1 i}}{\partial t}(t, x)=\kappa \Delta \mathbf{M}_{1 i}(t, x)+\frac{1}{2} \mathbf{M}_{1 i}(t, x), \quad i=1,2,3 .
$$

Since the spectrum of $\Delta$ is $[-4 d, 0]=[-12,0]$, for $d=3$, this equation implies that first moment Lyapunov exponent satisfies

$$
\gamma(1) \equiv \lim _{t \rightarrow \infty} \frac{1}{t} \log \left\|\mathbf{M}_{1}(t, x)\right\|=\frac{1}{2} .
$$

In [9], by simply using Itô's formula, an equation was derived for the average (over the media) magnetic field energy

$$
\mathbf{M}_{2}(t, x, y) \equiv E_{\mathbf{Q}}[\langle\mathbf{H}(t, x) \mathbf{H}(t, y)\rangle] .
$$

This satisfies

$$
\begin{aligned}
\frac{\partial \mathbf{M}_{2}}{\partial t}(t, x, y)= & \kappa\left(\Delta_{x}+\Delta_{y}\right) \mathbf{M}_{2}(t, x, y) \\
& +\mathbf{M}_{2}(t, x, y)+3 \delta_{x y} \mathbf{M}_{2}(t, x, y) .
\end{aligned}
$$

Under an assumption of homogeneity on $\mathbf{H}_{0}$, one has $\mathbf{M}_{2}(0, x, y)=\mathbf{m}_{2}(0, x-y)$ and so for all time $\mathbf{M}_{2}(t, x, y)=$ $\mathbf{m}_{2}(t, x-y)$, where

$$
\begin{aligned}
\frac{\partial \mathbf{m}_{2}}{\partial t}(t, x)= & 2 \kappa \Delta_{x} \mathbf{m}_{2}(t, x) \\
& +\mathbf{m}_{2}(t, x)+3 \delta_{0}(x) \mathbf{m}_{2}(t, x) .
\end{aligned}
$$

Writing $\mathbf{n}_{2}(t, x)=e^{-t} \mathbf{m}_{2}(t, x)$, this becomes

$$
\frac{\partial \mathbf{n}_{2}}{\partial t}(t, x)=2 \kappa \Delta_{x} \mathbf{n}_{2}(t, x)+3 \delta_{0}(x) \mathbf{n}_{2}(t, x) .
$$

In the physically relevant dimension $d=3$, taking the Fourier transform of the eigenvalue equation $2 \kappa \Delta_{x} \psi(x)+$ $3 \delta_{0}(x) \psi(x)=\lambda \psi(x)$ reveals that the operator $2 \kappa \Delta_{x}+3 \delta_{0}(x)$ possesses a positive eigenvalue $\lambda_{0}(\kappa)$ if and only if

$$
\frac{2 \kappa}{3}<\kappa_{c r} \equiv \int_{\mathrm{T}^{3}} \frac{d \phi}{4 \sum_{i=1}^{3}\left(1-\cos \phi_{1}\right)},
$$

where $\mathbf{T}^{3}$ is the three-dimensional torus. This implies that the solution $\mathbf{m}_{2}(t, x)$ satisfies

$$
\begin{gathered}
\gamma(2) \equiv \lim _{t \rightarrow \infty} \frac{1}{t} \log \mathbf{m}_{2}(t, x)=1, \quad \text { if } \frac{2 \kappa}{3} \geq \kappa_{c r}, \\
\gamma(2) \equiv \lim _{t \rightarrow \infty} \frac{1}{t} \log \mathbf{m}_{2}(t, x)=1+\lambda_{0}(\kappa), \quad \text { if } \frac{2 \kappa}{3} \leq \kappa_{c r} .
\end{gathered}
$$

This implies

$$
\gamma(2)=2 \gamma(1), \quad \kappa>\kappa_{c r}
$$

whereas

$$
\gamma(2)=\lambda_{0}(\kappa)+2 \gamma(1)>2 \gamma(1), \quad \kappa \leq \kappa_{c r} .
$$

This last inequality is the definition of full intermittency, and the second moment grows strictly faster than twice the first moment. As a consequence, the field $\mathbf{H}(t, x)$ has widely separated large peaks. This explains the well-known phenomena of sun spots. They are widely separated sites of high magnetic field energy. The main question of interest in astrophysics is whether the magnitude of $\mathbf{H}$ grows exponentially, a.s., in other words, is the a.s. Lyapunov exponent positive? This is the question of whether

$$
\lim _{t \rightarrow \infty} \frac{1}{t} \log \|\mathbf{H}(t, x)\|=\lambda(\kappa)>0 ?
$$

A further question regards the physically relevant asymptotic behavior of the a.s. Lyapunov exponent $\lambda(\kappa)$ as $\kappa \rightarrow 0$. Since $\kappa$ is the inverse Reynolds number, which in this situation is very small. Another interesting question is whether $e^{-t / 2} \mathbf{H}(t, x)$ has a limiting distribution. These questions have an affirmative answer in the scalar case and remain difficult open problems in the multidimensional model just discussed. 


\section{Parabolic Anderson Model}

The commonly studied parabolic Anderson equation is a scalar version of the magnetic field equation (1) and most progress has occurred in $\mathbf{Z}^{d}$ and we will treat this case first. The velocity field $\left\{v(t, x): t \geq 0, x \in \mathbf{R}^{d}\right\}$ may be replaced by a random environment that is either a stationary in time random field $\left\{\xi_{x}: x \in \mathbf{Z}^{d}\right\}$ or an evolving random field $\left\{\zeta_{x}(t): x \in \mathbf{Z}^{d}, t \geq 0\right\}$. Typically, the variables $\xi_{x}$ and $\zeta_{x}, x \in \mathbf{Z}^{d}$, in both cases are assumed to be iid. The stationary in time field can be thought of as a model where the phenomena of interest evolves much more rapidly than the evolution of the environment. The nonstationary case models a phenomena whose evolution is on a time scale comparable to the time scale of the evolution of the random environment. In this paper we will only discuss the nonstationary case. The enormous literature has been developed in this time stationary case. A partial random sample of works on this topic includes [12-17]. We will only discuss the nonstationary case. When considering the discrete model, that is, $\mathbf{Z}^{d}$, we take the operator $\Delta$ to be the discrete Laplacian as mentioned above, whereas it is the ordinary Laplacian when the model is set in $\mathbf{R}^{d}$. The parameter $\kappa>0$ in the model is called the diffusivity. The parabolic Anderson model is defined as a parabolic partial differential equation. A canonical version of this model is with the random environment provided by a white noise potential. In the $\mathbf{Z}^{d}$ case, one takes $\mathscr{W}=\left\{W_{x}: x \in\right.$ $\mathbf{Z}^{d}$ \} to be iid standard, one-dimensional Brownian motions defined on some probability space $\left(\Omega, \mathscr{F}_{t}, Q\right)$, where $\mathscr{F}_{t}=$ $\sigma\left\{W_{x}(s): x \in \mathbf{Z}^{d}, 0 \leq s \leq t\right\}$. This field is assumed to be $\delta$-correlated in time and space, that is, $E\left[W_{x}(t) W_{y}(s)\right]=$ $s \wedge t \delta_{0}(x-y)$ where $E$ denotes expectation with respect to $Q$. The differential form of the parabolic Anderson equation is then

$$
\begin{gathered}
d u(t, x)=\kappa \Delta u(t, x) d t+u(t, x) \partial W_{x}(t), \\
u(0, x)=u_{0}(x), \quad x \in \mathbf{Z}^{d}, t \geq 0 .
\end{gathered}
$$

The notation $\partial W_{x}(s)$ indicates the Stratonovich differential, (see [18] for a description of Stratonovich differentials) and this is preferred over the Itô differential because of the simplicity of the Feyneman-Kac representation which will appear shortly. The equivalent integral formulation is

$$
\begin{aligned}
u(t, x)= & u_{0}(x)+\kappa \int_{0}^{t} \Delta u(s, x) d s \\
& +\int_{0}^{t} u(s, x) \partial W_{x}(s), \quad x \in \mathbf{Z}^{d}, t \geq 0 .
\end{aligned}
$$

The differential formulation can be expressed converting to the Itô differential by

$$
\begin{aligned}
d u(t, x)= & \kappa \Delta u(t, x) d s+u(t, x) d W_{x}(s) \\
& +\frac{1}{2} u(t, x) d t \\
u(0, x)= & u_{0}(x), \quad x \in \mathbf{Z}^{d}, t \geq 0 .
\end{aligned}
$$

At times it will be convenient to discuss the Itô solution defined by

$$
\begin{gathered}
d u_{I}(t, x)=\kappa \Delta u_{I}(t, x) d s+u_{I}(t, x) d W_{x}(s), \\
u_{I}(0, x)=u_{0}(x), \quad x \in \mathbf{Z}^{d}, t \geq 0 .
\end{gathered}
$$

The relation between the two is that $u_{I}(t, x)=e^{-t / 2} u(t, x)$. Typically, it is assumed that $u_{0} \geq 0$ and that it is bounded from above, which guarantees existence and uniqueness of the solution. The positivity of $u_{0}$ assures that $u(t, x) \geq 0$ for all $(t, x) \in[0, \infty) \times \mathbf{Z}^{d}$. Fundamental information on this equation, including existence and uniqueness results, and its applications are contained in [19]. The field of solutions $\mathscr{U}(t) \equiv$ $\left\{u(t, x): x \in \mathbf{Z}^{d}\right\}$ exhibits interesting behavior as revealed by the growth properties of its moments which will be discussed at length below. At the risk of stating what is obvious, we stress that the random variables in the field $\left\{u(t, x): x \in \mathbf{Z}^{d}\right\}$ are dependent. The correlation structure of this field is examined in detail in Theorem 2 later in this paper.

The solution of (20) has a Feynman-Kac representation as an average over a path space. This is done by means of a family of measures $\left\{P_{x}^{\kappa}: x \in \mathbf{Z}^{d}\right\}$, on the set $\mathscr{D}([0, \infty)$, $\left.\mathbf{Z}^{d}\right) . \mathscr{D}\left([0, \infty), \mathbf{Z}^{d}\right)$ is the space of right-continuous paths possessing left limits from $[0, \infty) \rightarrow \mathbf{Z}^{d}$ which have a finite number of jumps on any compact subset of $[0, \infty)$. Define $\mathscr{D}_{T}=\mathscr{D}\left([0, T), \mathbf{Z}^{d}\right)$ analogously. We let $\left\{X_{t}: t \geq 0\right\}$ be the canonical process on $\mathscr{D}\left([0, \infty), \mathbf{Z}^{d}\right)$, that is, $X_{t}(\omega)=\omega(t)$ for $\omega \in \mathscr{D}\left([0, \infty), \mathbf{Z}^{d}\right)$. Then the measures $\left\{P_{x}^{\kappa}: x \in \mathbf{Z}^{d}\right\}$ are taken to be the ones that make $\left\{X_{t}, t \geq 0\right\}$ the pure jump Markov process on $\mathbf{Z}^{d}$ with generator $\kappa \Delta$. This process satisfies at $P_{x}^{\kappa}\left(X_{0}=x\right)=1$ and $X$ waits at $x$ for a random amount of time $\tau=\inf \left\{t: X_{t} \neq x\right\}$ which is exponentially distributed with parameter $2 d \kappa, P_{x}^{\kappa}(\tau \geq t)=e^{-2 d \kappa t}$, and then selects, using the uniform measure on its neighbors, one of the $2 d$ neighbors of $x$ and jumps there and then proceeds afresh as if starting from time 0 at the new position.

The solution to (20) can then be expressed as

$$
u(t, x)=E_{x}^{\kappa}\left[e^{\int_{0}^{t} d W_{X(t-s)}(s)} u_{0}\left(X_{t}\right)\right] .
$$

Unless explicitly mentioned otherwise, we will take $u_{0}(x) \equiv 1$ so that

$$
u(t, x)=E_{x}^{\kappa}\left[e^{\int_{0}^{t} d W_{X(t-s)}(s)}\right] .
$$

\section{Relations to Other Processes and Equations}

The parabolic Anderson equation is closely related to equations for many other models. Probably the best known connection to another equation is provided by the Hopf-Cole transformation. If we take a solution $u(t, x)$ to a parabolic Anderson equation

$$
\frac{\partial u}{\partial t}(t, x)=\kappa \Delta u(t, x)+F(t, x) u(t, x), \quad x \in \mathbf{R}^{d},
$$


with a spatially smooth random force term $F(t, x)$ and set $f(t, x)=\nabla F(t, x)$ and $v(t, x)=-2 \kappa \nabla \log u(t, x)$, then $v(t, x)$ satisfies Burger's equation

$$
\frac{\partial v}{\partial t}(t, x)+\langle v(t, x), \nabla\rangle v(t, x)=\kappa \Delta v(t, x)+f(t, x) .
$$

Similarly, setting $h=\log u$, where $u$ solves (23), yields a solution of the KPZ equation, see, for example, [4],

$$
\frac{\partial h}{\partial t}(t, x)=\kappa \Delta h(t, x)+\|\nabla h(t, x)\|^{2}+F(t, x) .
$$

Equation (20) may be cast as a particular case of a pair of equations driven by two iid jointly Gaussian, standard Brownian fields, $\left\{W_{t}^{i}(x): x \in \mathbf{Z}^{d}\right\}, i=1,2$ which also relates it to the mutually catalytic branching and stepping stone models. Assume the correlations between the fields is controlled by a parameter $h \in[-1,1]$ by

$$
E\left[W_{t}^{1}(x) W_{s}^{2}(y)\right]=h t \wedge s \delta_{0}(x-y)
$$

An interesting observation of Etheridge and Fleischmann, [20], neatly ties the symbiotic branching and stepping stone models together with the parabolic Anderson model by taking different values for the parameter $h$. Consider the following pair of equations:

$$
\begin{gathered}
d u(t, x)=\kappa \Delta u(t, x) d t+\sqrt{u(t, x) v(t, x)} d W_{x}^{1}(t), \\
d v(t, x)=\kappa \Delta v(t, x) d t+\sqrt{u(t, x) v(t, x)} d W_{x}^{2}(t), \\
u(0, x)=u_{0}(x), \quad v(0, x)=v_{0}(x) .
\end{gathered}
$$

When $h=0$ in (26), this is known as the mutually catalytic branching model for two interacting populations and has been studied in $[3,20-25]$ to name just a small random sample.

If $h=-1$ in (26) and $u_{0}(x)+v_{0}(x) \equiv 1$, then $u(t, x)+$ $v(t, x) \equiv 1, t \geq 0$ and $u(t, x)$ solves

$$
d u(t, x)=\kappa \Delta u(t, x) d t+\sqrt{u(t, x)(1-u(t, x))} d W_{x}^{1}(t)
$$

This is known as the stepping stone model from population genetics and has been the subject of the works $[2,16,26]$ among others.

Finally, when $h=1$ in (26) and $u_{0} \equiv v_{0}$ one gets $u(t, x) \equiv$ $v(t, x)$ and $u(t, x)$ is the solution of $(20)$ with $W_{x}(t)=W_{x}^{1}(t)$.

Other versions of noise have been considered as well and this can lead to substantial technical difficulties. For example, the recent works [27-31] replace the field $\mathscr{W}$ by catalysts which are from interacting particle systems such as the voter model or exclusion processes. Among the problems unresolved here is the existence of the a.s. Lyapunov exponent. The subadditive argument which worked so well for the environment $\mathscr{W}$ fails for these models.

\section{Moment Lyapunov Exponents}

One of the most interesting properties of the solutions of (20) is that of intermittency. Intermittency is defined in terms of the moment Lyapunov exponents. These are the limits for $p>$ 0 of

$$
\gamma_{p}(\kappa)=\lim _{t \rightarrow \infty} \frac{1}{t} \log E_{x}^{\kappa}\left[u^{p}(t, x)\right]
$$

The existence of these limits was proved in [19]. For $p=1$ one uses (116) to compute $\gamma_{1}(\kappa)$. This is easily done with the observation that for any fixed path $\{X(t-s): 0 \leq s \leq t\}$, the random variable

$$
A_{t}(X)=\int_{0}^{t} d W_{X(t-s)}(s)
$$

is Gaussian with mean 0 and variance $t$. Thus, by Fubini's theorem,

$$
E[u(t, x)]=e^{t / 2}
$$

so that $\gamma_{1}(\kappa)=1 / 2$. An interesting quantity, the overlap, arises when computing $\gamma_{2}(\kappa)$. Here we will use $E_{x}^{\kappa, \otimes 2}$ to denote the product measure of $E_{x}^{\kappa}$ with itself on $\mathscr{D}\left([0, \infty), \mathbf{Z}^{d}\right) \times$ $\mathscr{D}\left([0, \infty), \mathbf{Z}^{d}\right)$. Then we use the observation that for two fixed paths, $\{X(t-s): 0 \leq s \leq t\}$ and $\{Y(t-s): 0 \leq s \leq t\}$, the variables $A_{t}(X)$ and $A_{t}(Y)$ are jointly Gaussian with

$$
E\left[A_{t}(X) A_{t}(Y)\right]=\int_{0}^{t} \delta_{0}\left(X_{s}-Y_{s}\right) d s .
$$

Thus, $A_{t}(X)+A_{t}(Y)$ is Gaussian with mean 0 and variance

$$
\operatorname{var}\left(A_{t}(X)+A_{t}(Y)\right)=2\left(t+\int_{0}^{t} \delta_{0}\left(X_{s}-Y_{s}\right) d s\right)
$$

by (116),

$$
\begin{aligned}
E\left[u^{2}(t, x)\right] & =E\left[E_{x}^{\kappa, \otimes 2}\left[e^{A_{t}(X)+A_{t}(Y)}\right]\right] \\
& =E_{x}^{\kappa, \otimes 2}\left[E\left[e^{A_{t}(X)+A_{t}(Y)}\right]\right] \\
& =e^{t+\int_{0}^{t} \delta_{0}\left(X_{s}-Y_{s}\right) d s} .
\end{aligned}
$$

This implies

$$
\gamma_{2}(\kappa)=2 \gamma_{1}(\kappa)+\lim _{t \rightarrow \infty} \frac{1}{t} \log E_{x}^{\kappa, \otimes 2}\left[e^{\int_{0}^{t} \delta_{0}\left(X_{s}-Y_{s}\right) d s}\right] .
$$

For general $p \geq 1$, Hölder's inequality implies

$$
\frac{\gamma_{p}(\kappa)}{p} \leq \frac{\gamma_{p+1}(\kappa)}{p+1}, \quad \forall p \geq 1,
$$

and $p \rightarrow \gamma_{p}(\kappa)$ is convex. Full intermittency is then the property that $\gamma_{p}(\kappa)$ is strictly convex on $[1, \infty)$, that is,

$$
\frac{\gamma_{p}(\kappa)}{p}<\frac{\gamma_{p+1}(\kappa)}{p+1}, \quad \forall p \geq 1
$$


It is by now classical and was proven in [19], that in dimensions $d=1,2$, full intermittency holds for all $\kappa>0$ but in dimensions $d \geq 3$, full intermittency only holds for $0<$ $\kappa<\kappa_{2}(d)$ where $\kappa_{2}(d)$ is a dimension-dependent constant. This was done in [19] by noting that in dimensions $d=1$ and 2 the operator $2 \kappa \Delta+\delta_{0}$ has a positive eigenvalue for any $\kappa>0$. By contrast, in dimensions $d \geq 3$ there is a positive constant $\kappa_{2}(d)$ such that $2 \kappa \Delta+\delta_{0}$ has a positive eigenvalue only for $0<\kappa<\kappa_{2}(d)$. When there is a positive eigenvalue, $\lambda_{0}(\kappa)$, one can conclude that

$$
\lim _{t \rightarrow \infty} \frac{1}{t} \log E_{x}^{\kappa, \otimes 2}\left[e^{\int_{0}^{t} \delta_{0}\left(X_{s}-Y_{s}\right) d s}\right]=\lambda_{0}(\kappa) .
$$

When the spectrum of $2 \kappa \Delta+\delta_{0}$ has no eigenvalue, that is, when in dimension $d \geq 3$ with $\kappa>\kappa_{2}(d)$, one has

$$
\lim _{t \rightarrow \infty} \frac{1}{t} \log E_{x}^{\kappa, \otimes 2}\left[e^{\int_{0}^{t} \delta_{0}\left(X_{s}-Y_{s}\right) d s}\right]=0 .
$$

Using this with (120) we have the following consequence due to Carmona and Molchanov [19].

Theorem 1. Full intermittency holds in dimensions 1 and 2 for any $\kappa>0$. For each $d \geq 3$, there is a positive $\kappa_{2}(d)$ such that for $0<\kappa<\kappa_{2}(d)$, full intermittency holds.

Below we will give a probabilistic proof of this result which identifies the value $\kappa_{2}(d)$. In addition, in [19], it was shown that there is a sequence $\kappa_{p}(d)$ which was proved to be strictly increasing in [32], such that

$$
\begin{gathered}
\frac{\gamma_{p}(\kappa)}{p}=\gamma_{1}(\kappa), \quad \kappa \geq \kappa_{p}(d), \\
\frac{\gamma_{p}(\kappa)}{p}>\frac{\gamma_{p-1}(\kappa)}{p-1}, \quad \kappa<\kappa_{p}(d) .
\end{gathered}
$$

This has been refined, extended, and improved in the recent work of Greven and den Hollander, [32].

The physical phenomena of intermittency is the property that a random field possesses widely separated high peaks. The most well-known field exhibiting this property is the magnetic field energy in a star. In our sun, this exhibits itself as sun spots where most of the magnetic field energy is concentrated, thereby lowering the temperature and causing the darkening which appears as a spot. Intermittency properties of the field $\left\{u(t, x): x \in \mathbf{Z}^{d}\right\}$ were established in [19] and this will be discussed below.

\section{Itô Solution and Probabilistic Proof of Intermittency}

The Itô solution, $u_{I}(t, x)$, is the solution when the Itô differential is used in (20) instead of the Stratonovich differential. Recalling this solution satisfies

$$
\begin{aligned}
u_{I}(t, x)= & u_{0}(x)+\kappa \int_{0}^{t} \Delta u_{I}(s, x) d s \\
& +\int_{0}^{t} u_{I}(s, x) d W_{x}(s), \quad x \in \mathbf{Z}^{d}, t \geq 0 .
\end{aligned}
$$

The relation between $u$ and $u_{I}$ is simply

$$
u_{I}(t, x)=e^{-t / 2} u(t, x) .
$$

Then defining $\gamma_{2}^{I}(\kappa)=\lim _{t \rightarrow \infty}(1 / t) \log E\left[u_{I}^{2}(t, x)\right]$, it follows that

$$
\gamma_{2}(\kappa)-2 \gamma_{1}(\kappa)=\gamma_{2}^{I}(\kappa)
$$

and so full intermittency holds if and only if $\gamma_{2}^{I}(\kappa)>0$.

We show that in dimensions greater than 2 , that the Itô solution $u_{I}(t, x)$ has bounded second moment for $\kappa>\kappa_{2}(d)$ and that the second moment grows exponentially for $\kappa \leq$ $\kappa_{2}(d)$. This was shown in [19] using spectral considerations outlined in the previous section, so here we will give a probabilistic argument which was alluded to in [19] but does not seem to have appeared anywhere. First, recall that the jump rate of $X$ is $2 d \kappa$. Thus, if $Y$ is an independent copy of $X$, the jump rate of $X-Y$ is $4 d \kappa$, that is, $\left(\left\{\left(X_{s}-Y_{s}\right): s \geq\right.\right.$ $\left.0\}, P_{x}^{\kappa}\right) \stackrel{\mathscr{L}}{=}\left(\left\{X_{s}: s \geq 0\right\}, P_{x}^{2 \kappa}\right)$. Recalling that

$$
E\left[e^{A_{t}(X)+A_{t}(Y)}\right]=e^{t+\int_{0}^{t} \delta_{0}\left(X_{s}-Y_{s}\right) d s},
$$

we have

$$
\begin{aligned}
E\left[u_{I}^{2}(t, x)\right] & =e^{-t} E\left[E_{x}^{\kappa, \otimes 2}\left[e^{A_{t}(X)+A_{t}(Y)}\right]\right] \\
& =e^{-t} E_{x}^{\kappa, \otimes 2}\left[E_{\mathrm{Q}}\left[e^{A_{t}(X)+A_{t}(Y)}\right]\right] \\
& =E_{x}^{\kappa, \otimes 2}\left[e^{\int_{0}^{t} \delta_{0}\left(X_{s}-Y_{s}\right) d s}\right] \\
& =E_{x}^{2 \kappa}\left[e^{\int_{0}^{t} \delta_{0}\left(X_{s}\right) d s}\right] .
\end{aligned}
$$

Now we introduce some helpful notation. We let $T_{1}=$ $\tau_{1}=\inf \left\{t>0: X_{t} \neq X_{0}\right\}, \rho_{0}=0$ and for $j \geq 1$,

$$
T_{j}=\inf \left\{t>\rho_{j-1}: X_{t} \neq 0\right\}, \quad \rho_{j}=\inf \left\{t>T_{j}: X_{t}=0\right\} .
$$

If the underlying chain is recurrent, then all of these stopping times are finite. If the chain is transient, then only a finite number of these times is finite. Set, for $j \geq 1$,

$$
\tau_{j}=T_{j}-\rho_{j-1}, \quad U_{j}=\rho_{j}-T_{j} .
$$

The distribution of $\tau_{j}$ with respect to $P_{0}^{2 \kappa}$ is exponential with parameter $4 d \kappa$. For all $j \geq 1$, the random variable $\tau_{j}$ is the duration of the $j$ th sojourn at 0 and $U_{j}$ is the duration of the $j$ th excursion from 0 . In the recurrent case, the random variables $\tau_{1}, \tau_{2}, \ldots$ and $U_{1}, U_{2}, \ldots$ are all finite and independent. They are also independent in the transient case "up to the time they all become infinite." For a given $t$, if $T_{n} \leq t \leq T_{n+1}$, then writing $R_{t}=\int_{0}^{t} \delta_{0}\left(X_{s}\right) d s$,

$$
\tau_{1}+\cdots+\tau_{n} \leq R_{t} \leq \tau_{1}+\cdots+\tau_{n+1} .
$$

The skeletal random walk of $X$ can now be defined using these stopping times. This is the Markov chain on $\mathbf{Z}^{d}$ which 
keeps track of the sites visited by $X$, namely, $Y_{0}=X_{0}, Y_{n}=$ $X_{T_{n}+}, n \geq 1$. Observe that if $R=\sum_{n \geq 0} \delta_{0}\left(Y_{n}\right)$, then $R$ is a geometric random variable with parameter $r=P_{0}^{2 \kappa}(R=$ $1)$, the probability of no return to the origin which is independent of $\kappa$. For $d \geq 3$, and it is well known that $r>0$, see, for example, [2]. Since $R$ and the random variables $\tau_{n}$ are independent, an easy argument shows that the total time spent at the origin is thus

$$
\int_{0}^{\infty} \delta_{0}\left(X_{s}\right) d s=\sum_{n=1}^{R} \tau_{n}
$$

and is an exponentially distributed random variable with parameter $4 d \kappa r$ with respect to the measure $P_{0}^{2 \kappa}$. If $\kappa>1 / 4 d r$, one has

$$
\lim _{t \rightarrow \infty} E\left[u_{I}^{2}(t, 0)\right]=E_{0}^{2 \kappa}\left[e^{\sum_{n=1}^{R} \tau_{n}}\right]=\frac{4 d \kappa r}{4 d \kappa r-1}<\infty .
$$

We remark that by stationarity of the field $\mathscr{W}$ and the choice of $u_{0} \equiv 1$, it follows that $E\left[u_{I}^{2}(t, x)\right]$ is independent of $x$. When $\kappa \leq 1 / 4 d r$, one has $E\left[u_{I}^{2}(t, 0)\right]=\infty$. An important consequence of this little computation is the lack of intermittency for $\kappa>\kappa_{2, c r} \equiv 1 / 4 d r$. Indeed, for $\kappa>\kappa_{2, c r}=$ $1 / 4 d r$

$$
\lim _{t \rightarrow \infty} \frac{1}{t} \log E\left[u(t, x)^{2}\right]=\lim _{t \rightarrow \infty} \frac{1}{t} \log e^{t} E\left[u_{I}(t, x)^{2}\right]=1 .
$$

Consequently, since $\gamma_{1}(\kappa)=1$, for $\kappa>\kappa_{2, c r}=1 / 4 d r$,

$$
\gamma_{2}(\kappa)=2 \gamma_{1}(\kappa)
$$

and there is no intermittency for large $\kappa$ in dimensions $d \geq 3$.

For $d=1$ or 2 , however, we proceed as follows to show that there is intermittency for all $\kappa>0$. It will be useful to make a change of variable and to set up some more notation. First we observe that $\left(\left\{X_{s / 4 d \kappa}: s \geq 0\right\}, P_{x}^{2 \kappa}\right) \stackrel{\mathscr{L}}{=}\left(\left\{X_{s}: s \geq\right.\right.$ $\left.0\}, P_{x}^{1 / 2 d}\right)$. Thus,

$$
E_{x}^{2 \kappa}\left[e^{A_{t}(X)}\right]=E_{x}^{1 / 2 d}\left[e^{(1 / 4 d \kappa) A_{t}(X)}\right]
$$

We use Theorem 2 and it then suffices to show $\lim _{t \rightarrow \infty}(1 /$ t) $\log E_{0}^{1 / 2 d}\left[e^{(1 / 4 d \kappa) A_{t}(X)}\right]>0$. For simplicity, we denote

$$
\beta=\frac{1}{4 d \kappa}
$$

Notice that the random variables $\tau_{n}$ are exponentially distributed with parameter 1 with respect to $P_{0}^{1 / 2 d}$.

Recall for $d=1$ or 2 that $r=0$. Choose $K$ sufficiently large so that $P_{0}^{1 / 2 d}\left(U_{1} \leq K\right) \geq e^{-\beta / 2}$. Then,

$$
\begin{aligned}
P_{0}^{1 / 2 d}\left(U_{1}+\cdots+U_{n} \leq K n\right) & \geq P_{0}^{1 / 2 d}\left(\max \left\{U_{1}, \ldots, U_{n}\right\} \leq K\right) \\
& \geq e^{-\beta n / 2} .
\end{aligned}
$$

The central limit theorem implies that $P_{0}^{1 / 2 d}\left(\tau_{1}+\cdots+\tau_{n} \geq\right.$ $n) \geq 1 / 4$ for $n$ sufficiently large. Observe that

$$
\left\{\tau_{1}+\cdots+\tau_{n} \geq n, U_{1}+\cdots+U_{n} \leq K n\right\} \subset\left\{R_{n(K+1)} \geq n\right\} .
$$

Hence,

$$
\begin{aligned}
E_{0}^{1 / 2 d} & {\left[e^{\beta R_{n(K+1)}}\right] } \\
& \geq E_{0}^{1 / 2 d}\left[e^{\beta R_{n(K+1)}}: \tau_{1}+\cdots+\tau_{n} \geq n, U_{1}+\cdots+U_{n} \leq K n\right] \\
& \geq e^{\beta n} P_{0}^{1 / 2 d}\left(\tau_{1}+\cdots+\tau_{n} \geq n, U_{1}+\cdots+U_{n} \leq K n\right) \\
& \geq \frac{1}{4} e^{n / 2} \\
& =\frac{1}{4} e^{(\beta / 2(K+1))(K+1) n} .
\end{aligned}
$$

Consequently,

$$
\begin{aligned}
\gamma_{I}(2) & =\lim _{n \rightarrow \infty} \frac{1}{(K+1) n} \log E_{0}^{1 / 2 d}\left[e^{\beta R_{n(K+1)}}\right] \\
& \geq \frac{\beta}{2(K+1)} \\
& >0 .
\end{aligned}
$$

As a result, intermittency $\gamma_{2}(\kappa)>2 \gamma_{1}(\kappa)$ holds for all $\kappa>0$ in dimensions $d=1$ and 2 .

We return now to $d \geq 3$ so that $r>0$ and show that intermittency holds for $\kappa<1 / 4 d r$. Again, it suffices to show $\lim _{t \rightarrow \infty}(1 / t) \log E_{0}^{1 / 2 d}\left[e^{\beta A_{t}(X)}\right]>0$. Recall that the $\tau_{n}$ are exponential distributed with parameter 1 with respect to $P_{0}^{1 / 2 d}$. Standard large deviation estimates (see, e.g., [33, Theorem 9.3]) show that for fixed $\delta>0$,

$$
P_{0}^{1 / 2 d}\left(\tau_{1}+\cdots+\tau_{n} \geq(1+\delta) n\right) \approx e^{n(\log (1+\delta)-\delta)} .
$$

Also,

$$
P_{0}^{1 / 2 d}\left(\max \left\{U_{1}, \ldots, U_{n}\right\}<\infty\right)=e^{n \log (1-r)} .
$$

As in the previous paragraph, for every $\epsilon>0$ we can find a $K$ such that

$$
\begin{aligned}
P_{0}^{1 / 2 d} & \left(U_{1}+\cdots+U_{n} \leq K n\right) \\
& \geq P_{0}^{1 / 2 d}\left(\max \left\{U_{1}, \ldots, U_{n}\right) \leq K\right) \\
& =e^{n(\log (1-r)-\epsilon)} .
\end{aligned}
$$

Choosing $1+\delta=(1-r)^{-1}$, we get

$$
\begin{aligned}
& d P\left(\tau_{1}+\cdots+\tau_{n} \geq \frac{n}{1-r}, \max \left\{U_{1}, \ldots, U_{n}\right) \leq K\right) \\
& \approx e^{-(n /(1-r))(r+\epsilon(1-r))} .
\end{aligned}
$$


Recalling (56), it follows that for all $n$ sufficiently large,

$$
P\left(R_{n(K+1)} \geq(1 /(1-r)) n\right) \geq e^{-(n /(1-r))(r+\epsilon(1-r))} .
$$

If $\beta>r$ and we choose $\epsilon$ with $\epsilon<(\beta-r) /(4(1-r))$, with the corresponding choice of $K$ as in (61), then for all sufficiently large $m$,

$$
\begin{aligned}
E\left[e^{\beta R_{n(K+1)}}\right] & \geq e^{(\beta /(1-r)) n} e^{-(n /(1-r))(r+\epsilon(1-r))} \\
& \geq e^{((\beta-r-\epsilon(1-r)) /((1-r)(K+1))) n(K+1)} .
\end{aligned}
$$

Therefore, $\lim _{t \rightarrow \infty}(1 / t) \ln E_{0}^{1 / 2 d}\left[e^{\beta A_{t}(X)}\right] \geq(\beta-r-\epsilon(1-$ $r)) /((1-r)(K+1))$, for every $\epsilon>0$ and intermittency holds in dimension $d=1$ or 2 for all $\kappa>0$.

\section{Covariance Structure and Association}

Now we will examine the covariance structure of the field $\mathcal{U}(t)$ in the intermittent regime, that is, when $\kappa<\kappa_{2}(d)$ so that $2 \gamma_{1}(\kappa)<\gamma_{2}(\kappa)$. Recall that this range for $\kappa$ corresponds exactly to the range of $\kappa$ for which the operator $2 \kappa \Delta+\delta_{0}$ has a positive eigenvalue. We will also establish that this field is associated. The mixed second moments $E[u(t, x) u(t, y)]$ are significant in understanding the structure of the field $\mathscr{U}(t)$. They are given by

$$
\begin{aligned}
E[u(t, x) u(t, y)] & =e^{t} E_{x}^{\kappa} \otimes E_{y}^{\kappa}\left[e^{\int_{0}^{t} \delta_{0}\left(X_{s}-Y_{s}\right) d s}\right] \\
& =e^{t} E_{x-y}^{2 \kappa}\left[e^{\int_{0}^{t} \delta_{0}\left(X_{s}\right) d s}\right] .
\end{aligned}
$$

The asymptotics of the function $E[u(t, x) u(t, y)]$ can be evaluated as follows. Define

$$
Z_{\beta, t}^{\kappa}(x)=E_{x}^{\kappa}\left[e^{\beta \int_{0}^{t} \delta_{0}\left(X_{s}\right) d s}\right]
$$

Then observe that

$$
e^{-t} E[u(t, x) u(t, y)]=Z_{1, t}^{2 \kappa}(x-y) .
$$

But there is a scaling relation

$$
\begin{aligned}
Z_{1, t}^{2 \kappa}(x-y) & =E_{x-y}^{2 \kappa}\left[e^{\int_{0}^{t} \delta_{0}\left(X_{s}\right) d s}\right] \\
& =E_{x-y}^{2 \kappa}\left[e^{\int_{0}^{2 \kappa t} \delta_{0}\left(X_{s / 2 \kappa}\right) d(s / 2 \kappa)}\right] \\
& =E_{x-y}^{1}\left[e^{(1 / 2 \kappa) \int_{0}^{2 \kappa t} \delta_{0}\left(X_{s}\right) d s}\right] \\
& =Z_{1 / 2 \kappa, 2 \kappa t}^{1}(x-y)
\end{aligned}
$$

since $Y .=X_{(2 \kappa)^{-1}}$ is a rate $2 d$ simple symmetric random walk on $\mathbf{Z}^{\mathbf{d}}$ with respect to $P_{x}^{2 \kappa}$. This shows that

$$
Z_{1, t}^{2 \kappa}(x)=Z_{1 / 2 \kappa, 2 \kappa t}^{1}(x) .
$$

The function $Z_{\beta, t}(x) \equiv Z_{\beta, t}^{1}(x)$ arises as the partition function of a homopolymer, [34], and by the Feyman-Kac formula, $Z_{\beta, t}(x)$ solves

$$
\begin{gathered}
\frac{\partial}{\partial t} Z_{\beta, t}(x)=\Delta Z_{\beta, t}(x)+\beta \delta_{0}(x) Z_{\beta, t}(x), \\
Z_{\beta, 0}(x) \equiv 1 .
\end{gathered}
$$

The spectrum of the operator on the right hand side,

$$
H_{\beta}=\Delta+\beta \delta_{0},
$$

satisfies

$$
\begin{aligned}
\operatorname{spectrum} & \left\{H_{\beta}\right\} \\
= & {[-4 d, 0] \cup\left\{\lambda_{0}(\beta)\right\}, \quad \text { for } \beta>0, d=1,2, }
\end{aligned}
$$

and for $d \geq 3$, there is a dimension-dependent $\beta_{c}(d)$ such that

$$
\begin{aligned}
& \operatorname{spectrum}\left\{H_{\beta}\right\} \\
& =[-4 d, 0], \quad \text { for } 0<\beta<\beta_{c}(d), d \geq 3, \\
& \text { spectrum }\left\{H_{\beta}\right\} \\
& =[-4 d, 0] \cup\left\{\lambda_{0}(\beta)\right\}, \quad \text { for } \beta_{c}(d)<\beta, d \geq 3 .
\end{aligned}
$$

In the above, $\lambda_{0}(\beta)>0$ is a simple eigenvalue for $H_{\beta}$ and the portion $[-4 d, 0]$ is purely a.c. spectrum. In fact, one now sees that $\beta_{c}(d)=1 / 2 \kappa_{2}(d)$ from the section on intermittency. We denote by $\psi_{\beta}$ the eigenfunction corresponding to $\lambda_{0}(\beta)$ and note that it is given by, see [34],

$$
\psi_{\beta}(x)=\frac{1}{(2 \pi)^{d}} \int_{\mathrm{T}^{d}} \frac{e^{i\langle\phi, x\rangle}}{\lambda_{0}(\beta)+\Phi(\phi)} d \phi,
$$

where

$$
\Phi(\phi)=2 \sum_{j=1}^{d}\left(1-\cos \phi_{j}\right)
$$

is the symbol (Fourier transform) of $\Delta$ and $\mathbf{T}^{d}$ is the $d$ dimensional torus. The representation (74) can be used to establish the exponential decay of $\psi_{0}$, and there is a positive constant $c=c(\beta, d)$ such that

$$
\psi_{\beta}(x) \leq c e^{-c|x|}, \quad x \in \mathbf{Z}^{d} .
$$

By the spectral theorem, letting $E_{\lambda}$ be the resolution of the identity for the operator $H_{1 / 2 \kappa}$, one has

$$
\begin{aligned}
Z_{1 / 2 \kappa, t}(x)= & e^{\lambda_{0}(1 / 2 \kappa) t} \psi_{1 / 2 \kappa}(x)\left\|\psi_{1 / 2 \kappa}\right\|_{L^{1}} \\
& +\int_{-4 d}^{0} e^{\lambda t}\left\langle d E_{\lambda} 1_{x}, 1\right\rangle .
\end{aligned}
$$

Note that $Z_{1 / 2 \kappa, t}(x)=E_{x}^{1 / 2 \kappa}\left[e^{\int_{0}^{t} \delta_{0}(X(s)) d s}\right] \geq 1$. The following result was used in [19] to prove a central limit theorem for sum of the form $\sum_{x \in \Lambda_{L}} u(t, x)$ which will be described later in this paper. 
Theorem 2. For $d=1,2$ and any $\kappa>0$ or for $d \geq 3$ and $\kappa<\kappa_{2}(d)=1 / 2 \beta_{c}(d)$,

$$
\begin{aligned}
& Z_{1 / 2 \kappa, t}(x)-1 \\
&= \exp \left(\lambda_{0}\left(\frac{1}{2 \kappa}\right) t\right) \\
& \quad \times\left(\psi_{1 / 2 \kappa}(x)\left\|\psi_{1 / 2 \kappa}\right\|_{L^{1}\left(\mathbb{R}^{d}\right)}+q(x) O(\exp (-\epsilon t))\right), \\
& t \rightarrow \infty,
\end{aligned}
$$

where

$$
\sum_{x \in \mathbf{Z}^{d}} q(x)<\infty .
$$

In addition, when there is a positive eigenvalue for $H_{1 / 2 \kappa}$, this eigenfunction satisfies

$$
\psi_{1 / 2 \kappa}(x) \leq c e^{-c|x|}, \quad x \in \mathbf{Z}^{d}
$$

where $c=c(1 / 2 \kappa, d)>0$ depends on $d$ and $\kappa$.

This implies exponential decay in the spatial variable for the covariance of solutions of (20). Recalling the equivalence in law stated in (69) it follows from (78) that

$$
Z_{1, t}^{2 \kappa}(x) \sim e^{\lambda_{0}(1 / 2 \kappa) 2 \kappa t}\left(\psi_{1 / 2 \kappa}(x)\left\|\psi_{1 / 2 \kappa}\right\|_{L^{1}}+q(x) O\left(e^{-\epsilon 2 \kappa t}\right)\right) .
$$

Corollary 3. For $d=1,2$ and any $\kappa>0$ or for $d \geq 3$ and $0<\kappa<\kappa_{2}(d)$,

$$
\begin{aligned}
& \operatorname{Cov}(u(t, x), u(t, y)) \\
&=e^{t}\left(Z_{1,2 \kappa t}^{2 \kappa}(x-y)-1\right) \\
& \sim e^{t} e^{\lambda_{0}(1 / 2 \kappa) 2 \kappa t}\left(\psi_{1 / 2 \kappa}(x-y)\left\|\psi_{1 / 2 \kappa}\right\|_{L^{1}}\right. \\
& \\
&\left.+q(x-y) O\left(e^{-\epsilon 2 \kappa t}\right)\right),
\end{aligned}
$$

$$
\begin{aligned}
E\left[u^{2}(t, x)\right] & =e^{t}\left(Z_{1, t}^{2 \kappa}(0)-1\right) \\
& \sim e^{t} e^{\lambda_{0}(1 / 2 \kappa) 2 \kappa t}\left(\psi_{1 / 2 \kappa}(0)\left\|\psi_{1 / 2 \kappa}\right\|_{L^{1}}\right. \\
& \left.+q(0) O\left(e^{-\epsilon 2 \kappa t}\right)\right) .
\end{aligned}
$$

Consequently,

$$
\begin{aligned}
\gamma_{2}(\kappa) & =2 \gamma_{1}(\kappa)+\lambda_{0}\left(\frac{1}{2 \kappa}\right) 2 \kappa \\
& =1+\lambda_{0}\left(\frac{1}{2 \kappa}\right) 2 \kappa .
\end{aligned}
$$

Note that (84) gives a quantitative expression for the intermittency condition $\gamma_{2}(\kappa)>2 \gamma_{1}(\kappa)$. Since $\gamma_{1}(\kappa)=1 / 2$, we see that

$$
\gamma_{2}(\kappa)-2 \gamma_{1}(\kappa)=\lambda_{0}\left(\frac{1}{2 \kappa}\right) 2 \kappa
$$

We note that $\lambda_{0}(1 / 2 \kappa) \rightarrow 0$ as $\kappa \nearrow \kappa_{2}(d)$. Its rate of decay depends on the dimension.

An important property of the field $\mathscr{U}(t)$ is that the random variables in this field are associated. A collection of random variables $\left\{X_{k}, k \in S\right\}$, where $S$ is a countable set, is said to be associated if for any $d$ and coordinate-wise increasing functions $f, g: \mathbf{R}^{d} \rightarrow \mathbf{R}$, and any finite subcollection $X_{k_{1}}, X_{k_{2}}, \ldots, X_{k_{d}}$, it holds that

$$
\operatorname{Cov}\left(f\left(X_{k_{1}}, X_{k_{2}}, \ldots, X_{k_{d}}\right), g\left(X_{k_{1}}, X_{k_{2}}, \ldots, X_{k_{d}}\right)\right) \geq 0 \text {. }
$$

This notion was introduced in [35] and is of course related to the FKG inequality. One important aspect of this property was developed in [36] where Newman established a central limit theorem for the collection $\left\{X_{k}, k \in \mathbf{Z}^{d}\right\}$ under the assumptions that the $\left\{X_{k}, k \in \mathbf{Z}^{d}\right\}$ are associated, stationary and satisfy finite susceptibility

$$
\sum_{k \in \mathbf{Z}^{d}} \operatorname{Cov}\left(X_{0}, X_{k}\right)<\infty .
$$

Note that the bound provided by (82) implies that the field $\mathcal{U}(t)$ has finite susceptibility in the intermittent regime. A classical application of the Newman's central limit theorem is to take the $X_{k}=\sigma(k), \sigma \in\{0,1\}^{Z^{d}}$, the spins of a ferromagnetic stochastic Ising model and derive a central limit theorem for sums, $\sum_{k \in \Lambda} \sigma(k)$, over growing boxes $\Lambda \subset \mathbf{Z}^{d}$, with respect to a Gibbs state. The spins are correlated, but they possess the property of being associated and stationary with respect to the Gibbs state.

The solutions of the parabolic Anderson equation (20) are associated. The following result was established in [14]. The proof uses a result of Pitt, [37], which states that a necessary and sufficient condition for the associativity of a Gaussian vector is the point-wise nonnegativity of its correlation function. Since associativity is preserved by convergence in distribution, the result below is proved using a simple approximation procedure.

Theorem 4. Let $\left\{W_{x}: x \in \mathbf{R}^{d}\right\}$ be a field of iid, standard, one-dimensional Brownian motions on some probability space $(\Omega, Q)$. Then $\mathscr{U}(t)=\left\{u(t, x): x \in \mathbf{Z}^{d}\right\}$, and the field of solutions of (20) is associated.

\section{Almost Sure Lyapunov Exponents}

In the previous section we examined the behavior of the moments of $u(t, x)$. We now turn our attention to the a.s. behavior of the solution of (20); that is, we consider the a.s. Lyapunov exponent defined by

$$
\lambda(\kappa)=\lim _{t \rightarrow \infty} \frac{1}{t} \log u(t, x) .
$$

The existence of this limit was first established by Carmona and Molchanov in [19] in the case when either $u_{0}(x)=$ $\delta_{0}(x)$ or, more generally, when $u_{0}$ has compact support. The technique of proof used Liggett's subadditive ergodic 
theorem, [38]. The sub-additivity, when $u_{0}=\delta_{0}$, is an easy consequence of the Feynman-Kac representation (116)

$$
\begin{aligned}
u(t+r, 0)= & E_{0}^{\kappa}\left[e^{\int_{0}^{t+r} d W_{X(t+u-s)}(s)} \delta_{0}\left(X_{t+u}\right)\right] \\
\geq & E_{0}^{\kappa}\left[e^{\int_{0}^{t+r} d W_{X(t+r-s)}(s)} \delta_{0}\left(X_{t+r}\right) \delta_{0}\left(X_{t}\right)\right] \\
= & E_{0}^{\kappa}\left[e^{\int_{0}^{t} d W_{X(t-s)}(s)} \delta_{0}\left(X_{t}\right)\right] \\
& \times E_{0}^{\kappa}\left[e^{\int_{0}^{r} d W_{X(r-s)}(t+s)} \delta_{0}\left(X_{r}\right)\right] \\
\stackrel{\mathscr{L}}{=} & u(t, 0) u(r, 0) .
\end{aligned}
$$

The Markov property is used in going from line 2 to line 3 and this technique broke down at this step in the case of $u_{0} \equiv 1$. The latter case was established in [39] using a block argument from percolation theory. This type of block argument originated in [40]. Using the fact that time increments of the field $\mathscr{W}$ are independent over disjoint space-time blocks in $[0, \infty) \times \mathbf{Z}^{d}$, the proof established an oriented percolation scheme and applied a recurrence result from [41] for such schemes.

In view of the application to stellar magnetic fields, a significant aspect of $\lambda(\kappa)$ is its asymptotic behavior as $\kappa \searrow 0$. The exact asymptotics were established independently in and in [42].

The asymptotics are derived from the Feynman-Kac representation (116) through analysis of the Gaussian field $\left\{H_{T}(\zeta): \zeta \in \mathscr{D}_{T}\right\}$ where

$$
H_{T}(\zeta)=\int_{0}^{T} d W_{\zeta(s)}(s), \quad \zeta \in \mathscr{D}_{T} .
$$

This field places a natural metric $\rho$ on $\mathscr{D}_{T}$ by means of

$$
\begin{aligned}
\rho^{2}(\zeta, \eta) & =E\left[\left(H_{T}(\zeta)-H_{T}(\eta)\right)^{2}\right] \\
& =2\left(T-\int_{0}^{T} \delta_{0}(\zeta(s)-\eta(s)) d s\right) .
\end{aligned}
$$

The index set $\mathscr{D}_{T}$ of the field $\left\{H_{T}(\zeta): \zeta \in \mathscr{D}_{T}\right\}$ is too large from the point of view of the metric entropy, see [43] for an explanation of the metric entropy, induced by the metric $\rho$. Thus we restrict the index set by specifying the number of jumps of its elements $\zeta$ in the interval. So, using $N(\gamma, t)$ to denote the number of jumps of $\zeta$ in $[0, t]$ we can define the space of paths

$$
\mathscr{D}_{n, m}=\left\{\zeta \in \mathscr{D}_{n}: \zeta:[0, n] \longrightarrow \mathbf{Z}^{d}, N(\gamma, n)=m\right\} .
$$

The superadditive functional

$$
A_{n, m}=\sup _{\zeta \in \mathscr{D}_{n, m}} H_{n}(\zeta)
$$

is the supremum of a Gaussian field

$$
\left\{H_{n}(\zeta): \zeta \in \mathscr{D}_{n, m}\right\}
$$

indexed by the set $\mathscr{D}_{n, m}$. This set has a suitably bounded entropy, which, by a theorem of Fernique and Talagrand, implies $E\left[A_{n, n}\right] \leq C n$. This bound allows, by means of Liggett's subadditive ergodic theorem, the conclusion that there is a positive constant $\alpha$ such that

$$
\lim _{n \rightarrow \infty} \frac{A_{n, n}}{n}=\alpha, \quad \text { a.s. }
$$

An interesting and presumably difficult problem is to determine the proper scale $A_{n, n}-\alpha n$ in order to obtain a limit law. It is conjectured that $\left(A_{n, n}-\alpha n\right) / n^{2 / 3}$ should have a nontrivial limit law, possibly related to the Airy distribution. This conjecture comes from related results arising in random matrix theory such as $[44,45]$. Similarly, one would expect nontrivial limit laws for $(\log u(t, x)-\lambda(\kappa) t) / t^{2 / 3}$.

The asymptotics established in $[39,42]$ for $\lambda(\kappa)$ is that

$$
\lim _{\kappa \supset 0} \frac{\lambda(\kappa)}{\log (1 / \kappa)}=\frac{\alpha^{2}}{4} .
$$

These asymptotics are arrived at by decomposing the Feynman-Kac representation, (116),

$$
\begin{gathered}
u(n, x) \stackrel{\mathscr{L}}{=} \sum_{j=0}^{\infty} E_{x}^{\kappa}\left[e^{\int_{0}^{n} H_{X}(n)} \mid N(X, n)=j\right] \\
\times P_{x}^{\kappa}(N(X, n)=j),
\end{gathered}
$$

where $\stackrel{\mathscr{L}}{=}$ denotes equality in law (distribution.) Note that the only change has been to have the time direction be the same in both $W$ and $X$. The intuition now is that the conditional expectation in the $j$ th term should be nearly $e^{A_{n, j}}$. One quickly realizes that sum over $j \notin[a n, b n]$ for suitable choices of $a$ and $b$ is not significant, then only terms of the from $e^{A_{n, j n}}$ matter. But, by Brownian scaling, $A_{n, j n} \stackrel{\mathscr{L}}{=}(1 / \sqrt{j}) A_{j n, j n}$. Using this in (97) and simple large deviations results for the Poisson distributed $N(X, n)$ leads to an upper bound for the asymptotics of $\lambda(\kappa)$. The lower bound comes from looking at a particular path $X$ that dominates the FeynamnKac expectation and using similar estimates.

Thus, for small $\kappa, \lambda(\kappa) \ll \gamma_{1}(\kappa) \equiv 1 / 2$ which says the a.s. behavior is much smaller than the first moment behavior. This just reflects the fact that the expectation of $u(t, x)$ is dominated by large values of $u(t, x)$ which occur with small, but not too small, probability. This is related to the intermittency and will be examined in the section on sums over boxes below.

We would like to point out that $\kappa \rightarrow \lambda(\kappa)$ is an increasing function of $\kappa$. Also, since $E[u(t, x)]=e^{t / 2}$ one has $\lambda(\kappa) \leq 1 / 2$ for all $\kappa>0$. Moreover, it was pointed out in [19] that $\lambda(\kappa)=$ $\gamma_{1}(\kappa)$ for $\kappa \geq \kappa_{2}(d)$. The argument given there goes as follows. First define

$$
\lambda_{I}(\kappa)=\lim _{t \rightarrow \infty} \frac{1}{t} \log u_{I}(t, x)
$$

From the fact that $u(t, x)=e^{t / 2} u_{I}(t, x)$, it follows that $\lambda_{I}(\kappa)=$ $-1 / 2+\lambda(\kappa)$. Note for $\kappa \geq \kappa_{2}(d)$ that $E\left[u_{I}^{2}(t, x)\right] \leq C(d)<\infty$ 
and $E\left[u_{I}(t, x)\right]=1$. Thus, for every $\epsilon>0$, there is a $\delta>0$ such that $P\left(u_{I}(t, x)>\delta\right)>\epsilon$. This implies $\lambda_{I}(\kappa) \geq 0$. But obviously, $\lambda_{I}(\kappa) \leq 0$ so we conclude that for $\kappa \geq \kappa_{2}(d)$ one has $\lambda_{I}(\kappa)=0$ and, consequently, $\lambda(\kappa)=1 / 2$.

We end this section with a discussion of the relation between the a.s. Lyapunov exponent and the moment Lyapunov exponents from [39].

Theorem 5. We have the following:

$$
\lambda(\kappa)=\gamma_{0}^{\prime}(\kappa)
$$

where $(d / d p) \gamma_{p}(\kappa)=\gamma_{p}^{\prime}(\kappa)$

We give a brief sketch of the proof. In [46], it was shown that $\lambda(\kappa)=\gamma_{0-}^{\prime}(\kappa)$. In [39] the large deviation estimate for every $\epsilon>0$ there is a $c(\epsilon)>0$ such that

$$
Q\left(u \geq e^{(\lambda(\kappa)+\epsilon) t}\right) \leq e^{-c(\epsilon) t}
$$

was established by means of a block argument. Thus, for $p>$ 0 ,

$$
\begin{aligned}
E\left[u^{p}(t, x)\right]= & E\left[u^{p}(t, x): u(t, x) \leq e^{c(\epsilon) t}\right] \\
& +E\left[u^{p}(t, x): u(t, x)>e^{c(\epsilon) t}\right] \\
\leq & e^{p(\lambda(\kappa)+\epsilon) t}+E\left[u^{2 p}(t, x)\right]^{1 / 2} e^{-c(\epsilon) t / 2} .
\end{aligned}
$$

Thus, for every $\epsilon>0$,

$$
\gamma_{0+}^{\prime}(\kappa) \leq \lim _{p \searrow 0}\left\{\lambda(\kappa)+\epsilon, \frac{1}{2 p}\left(\gamma_{2 p}(\kappa)-\epsilon\right)\right\}=\lambda(\kappa)+\epsilon
$$

which gives $\gamma_{0}^{\prime}(\kappa)=\lambda(\kappa)$.

\section{Solution of PAM as Interacting Diffusion}

An interesting point of view regarding the solution of (20) was proposed in $[47,48]$ which grew out of work on the stepping stone model in [26]. In [48], Shiga and Shimizu, the authors view the entire field $\mathscr{U}_{I}(t)=\left\{u_{I}(t, x): x \in \mathbf{Z}^{d}\right\}$ as a Markov process in a subset of a particular $L^{1}$-space. In [47, $48]$ and the related works [32, 49], a more general underlying Markov process is used than the one with generator $\kappa \Delta$. However, for simplicity we will confine our discussion to the case where the operator appearing in (20) is $\kappa \Delta$. Take any summable collection of positive numbers $\left\{\gamma_{x}: x \in \mathbf{Z}^{d}\right\}$ that also satisfies for some positive $C$,

$$
\sum_{y:|y-x|=1} \gamma_{y} \leq C \gamma_{x}
$$

Then set for $p>0$,

$$
\mathscr{L}^{p}(\gamma)=\left\{\xi \in[0, \infty)^{\mathbf{Z}^{d}}: \sum_{\xi \in \mathbf{Z}^{d}} \xi_{x}^{p} \gamma_{x}<\infty\right\} \subset L^{p}(\gamma) .
$$

The space $\mathscr{L}^{1}(\gamma)$ is endowed with the product topology. We recall the following theorem of [48].
Theorem 6. Given $\mathscr{U}_{I}(0) \in \mathscr{L}^{1}(\gamma)$, the SDE (20) has a unique strong solution with $\left(\mathscr{U}_{I}(t)\right)_{t \geq 0} \subset \mathscr{L}^{1}(\gamma)$ and strongly continuous paths in $\mathscr{L}^{2}(\gamma)$ a.s. The process $\left(\mathscr{U}_{I}(t)\right)_{t \geq 0}$ is a Markov process on $\mathscr{L}^{1}(\gamma)$ with semigroup $S_{t}$ which satisfies

$$
S_{t} f-f=\int_{0}^{t} S_{u} L f d u
$$

for $f$ depending smoothly on only finitely many coordinates and where

$$
\begin{aligned}
L F(\xi)= & \kappa \sum_{x \in \mathbf{Z}^{d}}\left(\sum_{y \in \mathbf{Z}^{d}:|y-x|=1} \kappa\left(\xi_{y}-\xi_{x}\right)\right) \frac{\partial f}{\partial \xi_{x}} \\
& +\frac{1}{2} \sum_{x \in \mathbf{Z}^{d}} \xi_{x}^{2} \frac{\partial^{2} f}{\partial \xi_{x}^{2}} .
\end{aligned}
$$

If $\mathscr{U}_{I}(0) \in \mathscr{L}^{2}(\gamma)$ then $\left(\mathcal{U}_{I}(t)\right)_{t \geq 0} \subset \mathscr{L}^{2}(\gamma)$ and is a Feller diffusion.

One interesting aspect of this theorem is the door it opens into applying the techniques of diffusion processes to the process $\mathscr{U}_{I}$. So one can ask what are the invariant measures for the semigroup $S_{t}$ ? Since the components of $\mathscr{U}_{I}$ are interacting processes, it also brings the point of view of interacting particle systems onto the scene. In the latter field, characterization of all the $S_{t}$-invariant, shift invariant, and ergodic measures is a common theme. The reader is referred to [50] or [51] for examples and many references. One example of such a result is the following due to Shiga from [47]. To describe his results we introduce some notation and relevant objects. Denote by $\mathscr{P}\left(\mathscr{L}^{2}(\gamma)\right)$ the probability measures on $\left(\mathscr{L}^{2}(\gamma), \mathscr{B}\left(\mathscr{L}^{2}(\gamma)\right)\right)$ where $\mathscr{B}\left(\mathscr{L}^{2}(\gamma)\right)$ is the Borel field on $\mathscr{B}\left(\mathscr{L}^{2}(\gamma)\right)$. Denote by $S_{t}^{*}$ the action of the dual of $S_{t}$ on the space $\mathscr{P}\left(\mathscr{L}^{2}(\gamma)\right)$, that is, with $\langle\mu, f\rangle=$ $\int_{\mathscr{L}^{2}(\gamma)} f(x) \mu(d x)$

$$
\left\langle S_{t}^{*} \mu, f\right\rangle=\langle\mu, f\rangle, \quad f \in C_{b}\left(\mathscr{L}^{2}(\gamma)\right) .
$$

Then we can define the invariant measures for the process $\mathcal{U}_{I}$ by

$$
\mathscr{I}=\left\{\mu \in \mathscr{P}\left(\mathscr{L}^{2}(\gamma)\right): S_{t}^{*} \mu=\mu, t \geq 0\right\} .
$$

We can also consider the class of probability measures that are invariant with respect to the group of shifts $\tau_{x}, x \in \mathbf{Z}^{d}$,

$$
\mathscr{T}=\left\{\mu \in \mathscr{P}\left(\mathscr{L}^{2}(\gamma)\right): \tau_{x} \mu=\mu, x \in \mathbf{Z}^{d}\right\} .
$$

Let

$$
\mathscr{T}_{1}=\left\{\mu \in \mathscr{T}:\left\langle\mu,\left|\xi_{x}\right|\right\rangle\left\langle\infty, x \in \mathbf{Z}^{d}\right\}\right.
$$

Consider the initial configuration $\mathscr{U}_{I}(0)=\Theta$ which means all coordinates take on the value $\theta \in[0, \infty)$. View this as starting the process $\mathcal{U}_{I}$ with initial distribution $\delta_{\Theta}$. An important result of Shiga, with additions made by $[32,49]$, explains the asymptotic behavior of $\mathcal{U}_{I}$ in the nonintermittent regime, $\kappa>\kappa_{2}(d)$. 
Theorem 7. Assume $d \geq 3$ and $\kappa>\kappa_{2}(d)$. Then

(i) for each $\theta \in \mathbf{R}, \lim _{t \rightarrow \infty} S_{t}^{*} \delta_{\Theta}=v_{\theta}$ exists and $\left\langle v_{\theta}, \xi_{x}\right\rangle=\theta$ for $x \in \mathbf{Z}^{d}$

(ii) the set of extreme points of $\mathcal{S} \cap \mathscr{T}_{1}$ is exactly $\left\{v_{\theta}: \theta \in\right.$ $\mathbf{R}\}$. If $\mu \in \mathscr{T}_{1}$ is ergodic with respect to the group of translations $\left\{\tau_{x}: x \in \mathbf{Z}^{d}\right\}$ and $\left\langle\mu, \xi_{x}\right\rangle=\theta$, then

$$
\lim _{t \rightarrow \infty} S_{t}^{*} \mu=v_{\theta}
$$

(iii) the measure $v_{\theta}$ is associated.

We observe that the association of $v_{\theta}$ follows from the association of the field $\mathcal{U}_{I}(t)$ since this property is preserved by limits in distribution. As a complement, Shiga in [47] also gave the behavior of the field $\mathscr{U}_{I}$ when $d=1,2$ so that the process is in the intermittent regime. More recently, this was extended to $d \geq 3$ by Cox and Greven, [49], and then Greven and den Hollander, [32], into the intermittent region, $0<\kappa<\kappa_{2}(d)$. In this case, it turns out that the process $\mathcal{U}_{I}(t)$ dies out in the following sense that its law tends to the point mass on the configuration of all 0's. By this we mean the element $\underline{0} \in[0, \infty)^{Z^{d}}$ all of whose components are 0 . We denote the point mass on $\underline{0}$ by $\delta_{\underline{0}}$.

Theorem 8. If $d=1,2$ then for every $\kappa>0$ and every $\mu \in \mathscr{T}^{1}$ if the initial law of $\mathcal{U}_{I}$ is $\mu$ then

$$
\lim _{t \rightarrow \infty} \mathscr{L}\left(\mathscr{U}_{I}(t)\right)=\delta_{\underline{0}} .
$$

As pointed out in [32], one may take $\mu=\delta_{\underline{\Theta}}, \theta \in[0, \infty)$, in which case $E[u(t, x)]=\theta, x \in \mathbf{Z}^{d}, t \geq 0$. Thus, while the system is dying out, this implies that there are very high, widely separated peaks in the field $\mathcal{U}_{I}(t)$ for large values of $t$. This is the phenomena of intermittency.

\section{Intermittency and Sums over Boxes}

In an effort to quantify the intermittency effect, we consider sums of the field of solutions $\mathcal{U}(t)=\left\{u(t, x): t \geq 0, x \in \mathbf{Z}^{d}\right\}$ over boxes in $\mathbf{Z}^{d}$ that grow in size as $t \rightarrow \infty$. This is inspired as well by the developments of [52], where limit laws for sums of products of exponentials of nonnegative, iid random variables $\left\{V_{i j}\right\}$, namely, $\sum_{i=1}^{N(t)} e^{\beta \sum_{j=1}^{t} V_{i j}}$ were studied. Under a Cramér type condition, $E\left[e^{s V_{i j}}\right]<\infty$ for some $s>0$, a weak law of large numbers, central limit theorem, and convergence to stable laws was established for $\sum_{i=1}^{N(t)} e^{\beta \sum_{j=1}^{t} V_{i j}}$ under appropriate rates of growth of $N(t)$ and proper centerings and scalings. Earlier efforts in this direction for time-stationary models were done in $[53,54]$. The transition to this setting is provided by considering the Feynman-Kac representation of solutions of the parabolic Anderson model which resembles sums of the form

$$
\sum_{i=1}^{N(t)} E_{i}\left[e^{\beta \sum_{j=1}^{t} V_{x(t-j), j}}\right]
$$

with $x(j), \quad j \geq 0$ a random walk on $\mathbf{Z}$ started at $i$ under the measure $P_{i}$. Recall that the solution of the stochastic equation

$$
u(t, x)=1+\kappa \int_{0}^{t} \Delta u(s, x) d s+\int_{0}^{t} u(s, x) \partial W_{x}(s)
$$

is given by means of the Feynman-Kac formula

$$
u(t, x)=E_{x}^{\kappa}\left[e^{\int_{0}^{t} d W_{X(t-s)}(s)}\right] .
$$

Thus, the analog of (113) would be

$$
\frac{1}{\left|\Lambda_{L}\right|} \sum_{x \in \Lambda_{L}} u(t, x)
$$

where

$$
\Lambda_{L}=\left\{x \in \mathbf{Z}^{d}:\|X\| \leq L\right\},
$$

and $\left|\Lambda_{L}\right|$ is the number.

Two observations follow from our previous considerations. First, if $L$ is fixed while $t \rightarrow \infty$, then the existence of the a.s. Lyapunov exponent implies that

$$
\lim _{t \rightarrow \infty} \frac{1}{t} \log \left(\frac{1}{\left|\Lambda_{L}\right|} \sum_{x \in \Lambda_{L}} u(t, x)\right)=\lambda(\kappa) .
$$

We will refer to (118) as the quenched average. On the other hand, if $t$ remains fixed while $L \rightarrow \infty$, then the ergodic theorem implies

$$
\lim _{L \rightarrow \infty} \frac{1}{\left|\Lambda_{L}\right|} \sum_{x \in \Lambda_{L}} u(t, x)=E[u(t, x)] \sim e^{\gamma_{1}(\kappa) t} .
$$

We will refer to (119) as the annealed average. When $d \geq 3$ and $\kappa>\kappa_{2}(d)$ there is no discrepancy between the quenched and annealed averages since, as was shown above, $\lambda(\kappa)=$ $\gamma_{1}(\kappa)=1 / 2$. However, when $d=1$ or 2 or when $d \geq 3$ and $\kappa<\kappa_{2}(d)$, one has $\lambda(\kappa)<\gamma_{1}(d)$ and as a result there arises a discrepancy between the quenched and annealed averages. This discrepancy is a manifestation of intermittency and if we allow $L=L(t)$ to grow at an appropriate rate, we can begin to quantify how large a box $\Lambda_{L}$ must be in order to capture the large peaks in the field that are giving rise to the annealed average.

Recall that for $\kappa<\kappa_{2}(d)$, full intermittency of the field $\left\{u(t, x): x \in \mathbf{Z}^{d}\right\}$ occurs which was defined as

$$
2 \gamma_{1}(\kappa)<\gamma_{2}(\kappa)
$$

It is the condition (120) which is at work behind the scenes in [55] and the main result gives some information on the spread of the high peaks as $t \rightarrow \infty$. We refer the reader to a more comprehensive exposition on intermittency in Grimmett and Kesten [56].

We now define two critical values. First, for $p>0$, set

$$
\eta(p)=\frac{1}{d}\left(p \gamma_{p}^{\prime}(\kappa)-\gamma_{p}(\kappa)\right)
$$

Here, the derivative is with respect to $p$. The critical values are $\eta(1)$ and $\eta(2)$. Since $\gamma_{0}^{\prime}(\kappa)=\lambda(\kappa)$, we have that $\eta(0)=0$. Full intermittency implies that $\eta(1)<\eta(2)$. Among the results of [55] were the following. 
Theorem 9. Assume $u_{0} \equiv 1$ and that $\kappa>0$ if $d=1,2$ or that $\kappa \in\left(0, \kappa_{2}(d)\right)$ when $d \geq 3$.

Quenched Asymptotics. If $\lim _{t \rightarrow \infty}(1 / t) \log L(t)=0$, then

$$
\lim _{t \rightarrow \infty} \frac{1}{t} \log \left(\frac{1}{\left|\Lambda_{L}\right|} \sum_{x \in \Lambda_{L}} u(t, x)\right)=\lambda(\kappa), \quad \text { a.s. }
$$

Transition Range. If $L(t)=e^{\gamma t}$ with $0<\gamma<\eta(1)$, then

$$
\limsup _{t \rightarrow \infty} \frac{1}{t} \log \left(\frac{1}{\left|\Lambda_{L}\right|} \sum_{x \in \Lambda_{L}} u(t, x)\right) \leq \gamma^{\prime}(b)-d \eta(b), \quad \text { a.s. }
$$

Annealed Asymptotics. If $L(t)=e^{\gamma t}$ with $\gamma>\eta(1)$, then for every $\epsilon>0$,

$$
\lim _{t \rightarrow \infty} Q\left(\left|\frac{\left(1 /\left|\Lambda_{L}\right|\right) \sum_{x \in \Lambda_{L}} u(t, x)}{E_{Q}[u(t, 0)]}-1\right| \geq \epsilon\right)=0 .
$$

In the range $\gamma>\eta(1)$ with $L(t)=e^{\gamma t}$, we have

$$
\lim _{t \rightarrow \infty} \frac{1}{t} \log \left(\frac{1}{\left|\Lambda_{L}\right|} \sum_{x \in \Lambda_{L}} u(t, x)\right)=\gamma_{1}(\kappa), \quad \text { in probability. }
$$

This means that at time $t$, the box $\Lambda_{L}(t)$ with $L(t)=e^{\gamma t}$ and $\gamma>\eta(1)$ is large enough to capture the high peaks which yield the annealed asymptotics, while in the subexponential regime,

$$
\begin{array}{r}
\lim _{t \rightarrow \infty} \frac{1}{t} \log \left(\frac{1}{\left|\Lambda_{L}\right|} \sum_{x \in \Lambda_{L}} u(t, x)\right) \\
=\lambda(\kappa)<\gamma_{1}(\kappa), \quad \text { Q-a.s. }
\end{array}
$$

Thus, boxes of subexponential size do not contain high peals in the field $\mathcal{U}(t)$. Notice that at $p=0, \gamma_{0}^{\prime}(\kappa)-d \eta(0)=\gamma_{0}^{\prime}(\kappa)=$ $\lambda(\kappa)$ while at $p=1, \gamma_{1}^{\prime}(\kappa)-d \eta(1)=\gamma_{1}(\kappa)$. The curve $\gamma_{p}^{\prime}(\kappa)-$ $d \eta(p)=(1-p) \gamma_{p}^{\prime}(\kappa)+\gamma_{p}(\kappa)$ as $p$ goes from 0 to 1 describes the transition of $\lim _{t \rightarrow \infty}(1 / t) \log \left(\left(1 / \Lambda_{L}\right) \sum_{x \in \Lambda_{L}} u(t, x)\right)$ from the quenched asymptotics at $b=0$ to the annealed asymptotics at $b=1$. Loosely speaking, if we interpret $\gamma$ in $L(t)=e^{\gamma t}$ as a temperature, there is a transition from the low temperature phase at $\gamma=0$ where one "low energy state" dominates (i.e., the behavior of $\left(1 / \Lambda_{L}\right) \sum_{x \in \Lambda_{L}} u(t, x)$ is dominated by one summand) to the high temperature phase at $\gamma>\gamma(1)$ where there is averaging. Going further, for $\gamma>\eta(2)$, we will see in the next section that the central limit theorem holds. This can be seen as a manifestation of complete disorder.

\section{Association and Central Limit Theorem}

In a previous section we discussed the property of association for the $\mathcal{U}(t)$. This property also held for the limiting laws $v_{\theta}$ of the field $\mathscr{U}_{I}(t)$ which arose when the distribution of $\mathscr{U}_{0}$ was $\delta_{\theta}$.

A central limit theorem for sums of the elements of the field $\mathscr{U}(t)$ was established in [55]. This theorem took the form

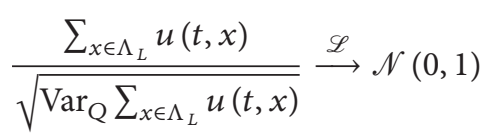

with $\Lambda_{L}=\left\{x \in \mathbf{Z}^{d}:\|X\|<L\right\}$ provided that $L=L(t)=e^{\gamma t}$ with $\gamma>\eta(2)$. The proof followed Bernstein's method of decomposing the sum (127) into sums over disjoint, slightly separated boxes. The proof in [55] was quite technical and relied on approximation of the solution $u(t, x)$ to obtain some degree of independence and a difficult large deviation result from [39]. An alternate proof of a stronger result, Theorem 11, using the ideas of Newman about associated random variables was given in [49]. The proof is simpler than the proof in [55] and yields more information about the variance of $\sum_{x \in \Lambda_{L}} u(t, x)$, relating it to the first eigenfunction of $2 \kappa \Delta+\delta_{0}$. The new proof also gives the joint distribution of these sums over disjoint growing boxes.

The key to the proof of a central limit theorem for associated random variables is the following inequality of [36].

Theorem 10 (Newman). Suppose $X_{1}, X_{2}, \ldots, X_{k}$ have finite variance and are associated. Then, for any $r_{1}, r_{2}, \ldots, r_{k} \in \mathbf{R}$,

$$
\begin{aligned}
& \left|E\left[e^{i \sum_{j=1}^{k} r_{j} X_{j}}\right]-\Pi_{j=1}^{k} E\left[e^{i r_{j} X_{j}}\right]\right| \\
& \quad \leq \frac{1}{2} \sum_{l \neq m}\left|r_{l}\right|\left|r_{m}\right| \operatorname{Cov}\left(X_{l}, X_{m}\right) .
\end{aligned}
$$

The content of this theorem is that if the sum of the covariances can be controlled, then the distribution of associated random variables can be compared to the distribution of independent random variables. The application of Newman's ideas only require an extension to triangular arrays of random variables and a verification of the finite susceptibility condition in Theorem 10.

Switching to $n$ to denote time, we will be concerned with sums of the variables $u(n, x)$ over boxes

$$
B_{k}^{n}=\left\{x \in \mathbf{Z}^{d}: k_{i} n \leq x_{i} \leq\left(k_{i}+1\right) n, i=1,2, \ldots, d\right\},
$$

for $k \in \mathbf{Z}^{d}$. The central limit result is then the following.

Theorem 11. Suppose $0<\kappa<\kappa_{c}(d)$ and let $\left\{u(n, j): j \in \mathbf{Z}^{d}\right\}$ be the solution of the parabolic Anderson equation (20). Define the random variables

$$
\begin{gathered}
X_{x}^{n}=e^{-(1 / 2) \gamma_{2}(\kappa) n} u(n, x), \quad x \in \mathbf{Z}^{d}, \\
Y_{k}^{m, n}=m^{-d / 2} \sum_{x \in B_{k}^{m}}\left(X_{x}^{n}-E\left[X_{x}^{n}\right]\right), \quad k \in \mathbf{Z}^{d}, \\
Y_{k}^{m(n)}=m(n)^{-d / 2} \sum_{x \in B_{k}^{m(n)}}\left(X_{x}^{n}-E\left[X_{x}^{n}\right]\right), \quad k \in \mathbf{Z}^{d} .
\end{gathered}
$$


If $m(n)=e^{\gamma n}$ with $\gamma>\eta(2)$, then

$$
Y_{k}^{m(n)} \stackrel{\mathscr{L}}{\longrightarrow} Z_{k}, \quad k \in \mathbf{Z}^{d},
$$

where the field

$$
\left\{Z_{k}: k \in \mathbf{Z}^{d}\right\}
$$

is composed of iid $\mathcal{N}(0, A)$ random variables with

$$
A=\left(\frac{1}{\lambda_{0}(1 / 2 \kappa)}\right)^{2}
$$

In order to verify the finite susceptibility condition, one uses (82), which gives

$$
\begin{aligned}
\sum_{y \in B_{0}^{n}} \operatorname{Cov}(u(n, 0), u(n, y)) & \\
& \sim e^{\gamma_{2}(\kappa) n} \sum_{y \in B_{0}^{n}}\left(\psi_{1 / 2 \kappa}(y)\left\|\psi_{1 / 2 \kappa}\right\|_{L^{1}}+q(y) O\left(e^{-\epsilon 2 \kappa n}\right)\right) .
\end{aligned}
$$

It then follows that

$$
\begin{aligned}
\sum_{y \in B_{0}^{n}} \operatorname{Cov}\left(X_{0}^{n}, X_{y}^{n}\right) & \\
& \sim \sum_{y \in B_{0}^{n}}\left(\psi_{1 / 2 \kappa}(y)\left\|\psi_{1 / 2 \kappa}\right\|_{L^{1}}+q(y) O\left(e^{-\epsilon 2 \kappa n}\right)\right) .
\end{aligned}
$$

Then, since

$$
\begin{aligned}
Y_{k}^{m(n)} & =m(n)^{-d / 2} \sum_{j \in B_{k}^{m(n)}}\left(X_{j}^{n}-E\left[X_{j}^{n}\right]\right) \\
& =m(n)^{-d / 2} \sum_{j \in B_{k}^{m(n)}}\left(X_{j}^{n}-e^{\left(\gamma_{1}(\kappa)-\gamma_{2}(\kappa) / 2\right) n}\right),
\end{aligned}
$$

one gets

$$
\begin{aligned}
& \operatorname{Var}\left(Y_{k}^{m(n)}\right) \\
& \left.=m(n)^{-d}\left(E\left[\sum_{x \in B_{k}^{m(n)}} X_{x}^{n}\right)^{2}\right]-m(n)^{2 d} e^{-n\left(\gamma_{2}(\kappa)-2 \gamma_{1}(\kappa)\right)}\right) \\
& \sim m(n)^{-d} \sum_{x, k \in B_{k}^{m(n)}}\left(\psi_{1 / 2 \kappa}(x-k)\left\|\psi_{1 / 2 \kappa}\right\|_{L^{1}}\right. \\
& \left.+m^{\prime}(n)^{d} e^{-n(\lambda(2)-2 \lambda(1))}(j-k) O\left(e^{-\epsilon 2 \kappa n}\right)\right) \\
& \sim \sum_{x \in B_{k}^{m(n)}}\left(\psi_{1 / 2 \kappa}(x)\left\|\psi_{1 / 2 \kappa}\right\|_{L^{1}}+q(x) O\left(e^{-\epsilon 2 \kappa n}\right)\right) \\
& -m(n)^{d} e^{-\lambda_{0}(\sqrt{2 \kappa}) 2 \kappa n} .
\end{aligned}
$$

Define

$$
A_{m(n)}=\operatorname{Var}\left(Y_{k}^{m(n)}\right)
$$

which, by stationarity, does not depend on $k \in \mathbf{Z}^{d}$ and

$$
A=\left(\sum_{j \in \mathbf{Z}^{d}} \psi_{1 / 2 \kappa}(j)\right)^{2} .
$$

By inverting the Fourier transform in (74), we have

$$
\widehat{\psi}_{1 / 2 \kappa}(\zeta)=\sum_{x \in \mathbf{Z}^{d}} \psi_{1 / 2 \kappa}(x) e^{-i\langle\zeta, x\rangle},
$$

so that by (74), we get

$$
\sum_{x \in \mathbf{Z}^{d}} \psi_{1 / 2 \kappa}(x)=\widehat{\psi}_{1 / 2 \kappa}(0)=\frac{1}{\lambda_{0}(1 / 2 \kappa)} .
$$

Then, by (137), one can conclude that $\lim _{n \rightarrow \infty} A_{m(n)}=$ $A$ which gives the variance of the limit in the theorem. Several open problems remain. The first question is whether a central limit theorem holds for a field with distribution $v_{\theta}$. This would only require checking the finite susceptibility condition and applying (87). Work similar to this is found in [57]. The second question is whether an invariance principle holds. There are invariance principles for associated fields established in [58-60]. Finally, one expects convergence of properly normalized and centered sums to a stable law of index $\alpha \in(0,2)$ when $L(t)=e^{\gamma t}$ and $\gamma=\eta(\alpha)$. Results that suggest this have appeared in $[52,61]$.

\section{Large Deviations and Concentration Effects}

In this section we examine some results on the distribution of the elements of the field $\mathscr{U}(t)$. We will begin with a concentration of measure result for an element of the field $\mathscr{U}(t)$. Early works on the concentration of measure phenomenon appeared in [62], for example. Recently, this subject has justifiably received a lot of attention, typical references might include [63-65] and the wonderful monograph of Ledoux [66], which has an extensive list of references. We recall an observation of Talagrand from [65]. The Chernoff bound for iid Bernoulli random variables $\epsilon_{i}$ states that

$$
P\left(\left|\sum_{i=1}^{n} \epsilon_{i}\right| \geq t\right) \leq 2 \exp \left\{-\frac{t^{2}}{2 N}\right\} .
$$

Talagrand's observation is "a random variable that smoothly depends on the influence of many independent random variables satisfies Chernoff-type bounds." The solution of (20), $u(t, x)$, depends smoothly on many independent random variables, namely, the increments in the Brownian field $\mathscr{W}(t) \equiv\left\{W_{x}(s): x \in \mathbf{Z}^{d}, 0 \leq s \leq t\right\}$. In order to make sense of this, it is natural to make use of the Malliavin calculus. Perhaps the first use of Mallaivin in disordered systems appeared in [67]. In the context of solutions of (20), Rovira and Tindel, [68], used the Malliavin calculus to establish the concentration inequality. 
The use of the Malliavin calculus, see [69] for more details, starts by expressing the functional, for a fixed path $X$,

$$
H_{T}(X)=\sum_{x \in \mathbb{Z}^{d}} \int_{0}^{T} d W_{x}(t) \delta_{x}(X(t))
$$

in the form

$$
W(h)=\sum_{x \in \mathbb{Z}^{d}} \int_{0}^{T} h(t, x) d W_{x}(t),
$$

where $h(t, x)$ depends on $X$ by the relation $h(t, x)=\delta_{x}(X(t))$. Obviously, $h \in L^{2}\left([0, T] \times \mathbb{Z}^{d}\right)$. The family $\{W(h): h \in$ $\left.L^{2}\left([0, T] \times \mathbb{Z}^{d}\right)\right\}$ is called a centered, isonormal Gaussian family and defines an abstract Wiener space as in [69]. The Malliavin derivative $D F$ of a square integrable random variable $F$ defined on this space is, when it exists, a random element of $L^{2}\left([0, T] \times \mathbb{Z}^{d}\right)$ that we will view as a stochastic process $D F=\left(D_{t, x} F\right)_{t, x}$ indexed by time and space. The Malliavin derivative $D_{t, x}$ is heuristically equal to $\partial / \partial\left(d W_{x}(t)\right)$ and can be formally computed as such. The Malliavin derivative of $H_{T}(X)$ is thus the element of $L^{2}\left([0, T] \times \mathbb{Z}^{d}\right)$ defined by

$$
D_{t, x} H_{T}(X)=\delta_{x}(X(t)) .
$$

Then taking $f(y)=e^{y}$ and applying the chain rule, we find the Malliavin derivative of $f\left(H_{T}(X)\right)$ is given by

$$
D_{t, x} f\left(H_{T}(X)\right)=f\left(H_{T}(X)\right) \delta_{x}(X(t)) .
$$

Setting $v(T, x)=E_{x}^{\mathcal{K}}\left[e^{H_{T}(X)}\right]$, and then differentiating yields

$$
D_{t, x} v(T, x)=E_{\kappa}\left[\delta_{x}(X(t)) \exp H_{T}(X)\right] .
$$

Using again the chain rule, we obtain

$$
D_{t, x} \log v(T, x)=\mu_{T}\left(\delta_{x}(X(t))\right),
$$

where $\mu_{T}$ is the probability measure on $\mathscr{D}_{T}$ defined by

$$
\mu_{T}(A)=\frac{E\left[1_{A} e^{H_{T}(X)}\right]}{v(T, X)} .
$$

Notice then that

$$
D_{t, x} \frac{1}{T} \log v(T, x) \leq \frac{1}{T}, \quad \text { a.s. }
$$

Also important is the bound

$$
\begin{aligned}
\|D \log v(T, x)\|_{L^{2}\left([0, T] \times \mathbb{Z}^{d}\right)}^{2} & =\beta^{2} \int_{0}^{T} \mu_{T}^{\otimes 2}(X(t)=Y(t)) d t \\
& \leq T .
\end{aligned}
$$

Thus

$$
\left\|D\left(\frac{1}{T} \log v(T, x)\right)\right\|_{L^{2}\left([0, T] \times \mathbb{Z}^{d}\right)}^{2} \leq 1 .
$$

Thus the functional $(1 / T) \log v(T, x)$ of the Brownian field $\mathscr{W}(T)$ is in the space $D^{1,2}$ of $L^{2}$-functionals whose first derivative is in $L^{2}$. This allows the application of a result from [70]: if $F \in D^{1,2}$ with $m=E[F]$ and $\sigma^{2}=\|D F\|_{\infty}$, for any $x>0$,

$$
P(|F-m| \geq x) \leq 2 \exp \left\{-\frac{x^{2}}{2 \sigma^{2}}\right\} .
$$

The application of this result, cited in [68], is the concentration inequality

$$
\begin{aligned}
& Q\left(\left|\frac{1}{T} \log v(T, x)-E\left[\frac{1}{T} \log v(T, x)\right]\right| \geq x\right) \\
& \quad \leq \exp \left\{-\frac{x^{2} T}{2}\right\} .
\end{aligned}
$$

Since $v(T, x) \stackrel{\mathscr{L}}{=} u(T, x)$, the concentration inequality

$$
\begin{aligned}
& Q\left(\left|\frac{1}{T} \log u(T, x)-E\left[\frac{1}{T} \log u(T, x)\right]\right| \geq x\right) \\
& \quad \leq \exp \left\{-\frac{x^{2} T}{2}\right\}
\end{aligned}
$$

is seen to hold for $u(T, X)$ as well. Thus, $(1 / T) \log u(T, x)$ has sub-Gaussian tails. Now as we have seen, $\lim _{t \rightarrow \infty}(1 /$ t) $\log u(t, x)=\lambda(\kappa)$ holds a.s. so one can conclude from (155) that

$$
\lim _{t \rightarrow \infty} \frac{1}{t} E[\log u(t, x)]=\lambda(\kappa) .
$$

The concentration results are thus closely related to large deviation results established in [39, 71-73]. For example, by means of a block argument, it was shown in [39] that for every $\epsilon>0$ there is a $c(e)>0$ such that

$$
Q\left(\frac{1}{t} \log u(t, x) \geq \lambda(\kappa)+\epsilon\right) \leq e^{-c(\epsilon) t} .
$$

This is not as precise as the bound in (155) which gives the upper bound $2 \exp \left\{-\epsilon^{2} t / 2\right\}$. An interesting effect here is that the upper bound for $Q((1 / t) \log u(t, x) \geq \lambda(\kappa)+\epsilon)$ is of the same order of magnitude in $t$ as the lower bound, but the probability of the event $\{(1 / t) \log u(t, x) \leq \lambda(\kappa)-\epsilon\}$ has much smaller order. The first point is verified by simply noting

$$
\begin{gathered}
Q\left(\frac{1}{t} \log u(t, x) \geq \lambda(\kappa)+\epsilon\right) \\
\quad \geq Q\left(W_{x}(t) \geq(\lambda(\kappa)+\epsilon) t\right) e^{-2 d \kappa t} \\
\leq c e^{-\left(2 d \kappa+(\lambda(\kappa)+\epsilon)^{2} / 2\right) t} .
\end{gathered}
$$

The probability of lower deviations for $(1 / t) \log u(x, t)$ below $\lambda(\kappa)-\epsilon$ has a much smaller order of magnitude than the probability for deviations above $\lambda(\kappa)+\epsilon$. This is similar to phenomena found in the first passage percolation [74] or in increasing subsequences in iid samples as in [75], to 
mention just a couple of instances. However, in contrast to the present situation, in the cited examples, the random variables involved have been positive. Since the model in [74] is close to the present case, we will give a brief description of the first passage percolation model and refer the reader to $[16,56,76$, 77]. The functionals involved in the first passage percolation stand in close analogy to the functional $A_{n, n}$ in Section 7. In first passage percolation on $\mathbf{Z}^{d}$ the edge set $\mathscr{E}$ is the set of edges between adjacent vertices in the usual lattice structure. The edges are endowed with an iid random field $\{t(e): e \in \mathscr{E}\}$ of non-negative random variables with common distribution function $F$. The random variable $t(e)$ assigned to an edge is thought of as the time required to traverse the edge $e$. One then considers "up-right" paths in the lattice, that is, sequences $\gamma=\left(v_{0}, e_{1}, v_{1}, e_{2}, \ldots, e_{n}, v_{n}\right)$ of alternating vertices and edges, where $v_{i}$ and $v_{i+1}$ are connected by the edge $e_{i}$ and the components of $v_{i+1}$ are greater than or equal to the components of $v_{i}$. The passage time of $\gamma$ is

$$
t(\gamma)=\sum_{i=1}^{n} t\left(e_{i}\right) .
$$

Then in [56] it was shown that the functional

$$
\begin{gathered}
\phi_{0, n}=\inf \{t(\gamma): \gamma \text { is an up-right path from } \\
\left.0 \in \mathbf{Z}^{d} \text { to }(n, n, \ldots, n) \in \mathbf{Z}^{d}\right\}
\end{gathered}
$$

which satisfies

$$
\lim _{n \rightarrow \infty} \frac{1}{n} \phi_{0, n}=\mu=\mu(F) .
$$

It was also observed there that $\mu(F)=0$ if and only if $F(0) \geq$ $p_{c}$ where $p_{c}$ is the critical probability for the existence of an infinite cluster in Bernoulli percolation in $\mathbf{Z}^{d}$. By means of a sub-additive argument, Chow and Zhang [74] showed that

$$
\lim _{n \rightarrow \infty} \frac{-1}{n} \log P\left(\phi_{0, n} \leq n(\mu-\epsilon)\right)=\alpha(\epsilon, F),
$$

whereas

$$
\liminf _{n \rightarrow \infty} \frac{-1}{n^{d}} \log P\left(\phi_{0, n} \geq n(\mu+\epsilon)\right)>0
$$

The intuitive reason for the difference is that for the event $\left\{\phi_{0, n} \geq n(\mu+\epsilon)\right\}$ to occur, the entire media need to be anomalous. However, for the event $\left\{\phi_{0, n} \leq n(\mu-\epsilon)\right\}$ to occur, the media only need to be anomalous along a single path from $0 \in \mathbf{Z}^{d}$ to $(n, n, \ldots, n)$.

In [73], a similar phenomenon was pointed out for the functional $A_{n, n}$ defined in (93). The limit (95) holds as in the case of the first passage percolation. There are two differences: the first is the rather minor one that the functional $A_{n, n}$ involves a sup rather than an inf. This has the effect of switching the roles of upper versus lower deviations. Now the event $\left\{A_{n, n} \geq(\alpha+\epsilon) n\right\}$ can occur if the media are anomalous along one path, whereas $\left\{A_{n, n} \leq(\alpha-\epsilon) n\right\}$ can only occur if the entire media up to time $n$ are anomalous. The fact that the random variables involved in the functional $A_{n, n}$ can be negative. However, due to the fact that the negative tails are not too heavy, this difficulty can be overcome and the following result holds.

Theorem 12. For $A_{n, n}$ as defined above in Model 1 in dimension $d$ and $\epsilon>0$, for the lower large deviations one has

$$
\begin{aligned}
-\infty & <\varliminf_{n \rightarrow \infty} \frac{1}{n^{d+1}} \log Q\left(\frac{A_{n, n}}{n} \leq \alpha-\epsilon\right) \\
& \leq \varlimsup_{n \rightarrow \infty} \frac{1}{n^{d+1}} \log Q\left(\frac{A_{n, n}}{n} \leq \alpha-\epsilon\right) \\
& <0,
\end{aligned}
$$

whereas for the upper large deviations, One has

$$
\begin{aligned}
-\infty & <\varliminf_{n \rightarrow \infty} \frac{1}{n} \log Q\left(\frac{A_{n, n}}{n} \geq \alpha+\epsilon\right) \\
& \leq \varlimsup_{n \rightarrow \infty} \frac{1}{n} \log Q\left(\frac{A_{n, n}}{n} \geq \alpha+\epsilon\right) \\
& <0 .
\end{aligned}
$$

An analogous result was established in [72] for solutions of (20). Generalizations of these results appeared in [71].

Theorem 13. For Model 1 and each $\epsilon>0$ for the lower large deviations, One has

$$
\begin{aligned}
-\infty & <\varliminf_{t \rightarrow \infty} \frac{1}{t^{d+1}} Q\left(\frac{1}{t} \log u(x, t) \leq \lambda(\kappa)-\epsilon\right) \\
& \leq \varlimsup_{t \rightarrow \infty} \frac{1}{t^{d+1}} Q\left(\frac{1}{t} \log u(x, t) \leq \lambda(\kappa)-\epsilon\right) \\
& <0,
\end{aligned}
$$

and for the upper large deviations, One has

$$
\begin{aligned}
-\infty & <\varliminf_{t \rightarrow \infty} \frac{1}{t} Q\left(\frac{1}{t} \log u(x, t) \geq \lambda(\kappa)+\epsilon\right) \\
& \leq \varlimsup_{t \rightarrow \infty} \frac{1}{t} Q\left(\frac{1}{t} \log u(x, t) \geq \lambda(\kappa)+\epsilon\right) \\
& <0 .
\end{aligned}
$$

\section{Parabolic Anderson in $R^{d}$}

The situation becomes technically more difficult when one considers the $\mathbf{R}^{d}$ version of (20). For the $\mathbf{R}^{d}$ model we let $\left\{W_{x}(t): x \in \mathbf{R}^{d}\right\}$ be a Gaussian field of identically distributed standard Brownian motions, defined on a probability space $(\Omega, F, Q)$. We can no longer assume that the motions are independent and obtain a solution to the analog of (20). The dependence will need to be spatially smooth for this so denote the correlation function of the field by $\Gamma(z)$,

$$
\Gamma(z) t \wedge s=E\left[W_{x}(t) W_{y}(s)\right], \quad z=x-y .
$$


Notice that we have the symmetry $\Gamma(z)=\Gamma(-z)$. We will assume that $\Gamma$ is continuously differentiable with first derivative Holder continuous of order $\beta-1$ for some $\beta>1$. The normalization $\Gamma(0)=1$ is forced by the assumption that $W$ is a standard Brownian motion. This gives the important approximation

$$
\Gamma(z)=1-\gamma|z|^{\beta}+o\left(|z|^{\beta}\right), \quad|z| \longrightarrow 0 .
$$

It is necessary to assume that $\gamma>0$ in order to avoid the degenerate case $\Gamma \equiv 1$.

The continuous space version of (20) in integrated form is thus

$$
\begin{aligned}
u(t, x)= & u_{0}(x)+\frac{\kappa}{2} \int_{0}^{t} \Delta u(s, x) d s \\
& +\int_{0}^{t} u(s, x) \partial W_{x}(s), \quad x \in \mathbf{R}^{d}, t>0,
\end{aligned}
$$

where $\Delta$ denotes the Laplacian in $\mathbf{R}^{d}$.

Equation (170) is called the parabolic Anderson model in $\mathbf{R}^{d}$. As noted in [78], unless the function $\Gamma(z)$ is twice continuously differentiable, (170) will not have a solution in that any prospective solution would lack a well-defined spatial Laplacian. Accordingly, the equation was reformulated as the integral equation

$$
\begin{array}{r}
u(t, x)=u_{0}(x) \\
+\int_{\mathbf{R}^{d}}\left[\int_{0}^{t} p(t-s, x, y) W_{y}(d s) u(s, y)\right] d y, \\
x \in \mathbf{R}^{d}, t>0,
\end{array}
$$

for $p(t-s, x, y)$ the Gausian kernel corresponding to speed $\kappa$ Brownian motion. This equation has the same solution as (170) in the case of a sufficiently smooth field $\left\{W_{x}: x \in\right.$ $\left.\mathbf{R}^{d}\right\}$. In [78], existence of solutions and other results was established. In particular, the Feynman-Kac representation remains valid for the following solution:

$$
u(t, x)=E_{x}^{\kappa}\left[e^{\int_{0}^{t} d W_{X(t-s)}(s)} u_{0}(X(t))\right]
$$

Here the expectation is taken with respect to $\{X(s): s \geq$ $0\}$ a speed $\kappa d$-dimensional Brownian motion, that is, the diffusion with generator $(\kappa / 2) \Delta$.

The principal result of [79] is the existence of the a.s. Lyapunov exponent for (170). The subadditivity results which worked so readily in the discrete spatial setting do not easily carry over to the present case. This technical difficulty can be overcome by a probabilistic version of a parabolic Harnack inequality.

Theorem 14. There exists a positive constant $\lambda(\kappa)$ such that for any nonnegative bounded function $u_{0}$ on $\mathbf{R}^{d}$ with $u_{0}>0$ on a set of strictly positive Lebesgue measure in $\mathbf{R}^{d}$, the solution $u$ of (170) with $u(0, \cdot)=u_{0}(\cdot)$ satisfies for any $x \in \mathbf{R}^{d}$,

$$
\lim _{t \rightarrow \infty} \frac{1}{t} \log u(t, x)=\lambda(\kappa) \quad \text { Q-a.s. }
$$

The $\kappa \rightarrow 0$ asymptotics are of a different order of magnitude in $\mathbf{R}^{d}$ than in $\mathbf{Z}^{d}$. In [79] it was shown that

$$
c \kappa^{1 / 3} \leq \lambda(\kappa) \leq C \kappa^{1 / 5}, \quad \kappa \longrightarrow 0 .
$$

However, recently, Rael [80] has shown that $\kappa^{1 / 3}$ is the correct order of magnitude,

$$
c \kappa^{1 / 3} \leq \lambda(\kappa) \leq C \kappa^{1 / 3}, \quad \kappa \longrightarrow 0 .
$$

It is an open problem to show that for some constant $\eta>0$,

$$
\lim _{\kappa \rightarrow 0} \kappa^{-1 / 3} \lambda(\kappa)=\eta
$$

\section{Anderson Polymer Models}

The field $\mathscr{W}(T)$ can be viewed as a random media through which the process $\left(X, P_{x}^{\kappa}\right)$ will evolve up to time $T$. The influence of the media on the process is obtained by a change of measure. The resulting measure on $\mathscr{D}_{T}$ is viewed as a measure on polymers. The Anderson polymer model is the Gibbs measure on $\mathscr{D}_{T}=\mathscr{D}\left([0, T], \mathbf{Z}^{d}\right)$ defined by

$$
\mu_{\kappa, \beta, T}^{x}(f)=Z_{\kappa, \beta, T}(x)^{-1} E_{x}^{\kappa}\left[f \exp \left\{\beta \int_{0}^{T} d W_{X(s)}(s)\right\}\right]
$$

for bounded measurable $f: \mathscr{D}_{T} \rightarrow \mathbb{R}$, where $Z_{\kappa, \beta, T}(x)=$ $E_{x}^{\kappa}\left[\exp \left\{\beta \int_{0}^{T} d W_{X(s)}(s)\right\}\right]$ is the partition function. This model has received a lot of attention in recent years and a partial list of references would include [33, 68, 81-95].

By the Feynman-Kac formula,

$$
u_{\beta}(t, x)=E_{x}^{\kappa}\left[\exp \left\{\beta \int_{0}^{t} d W_{X(t-s)}(s)\right\}\right]
$$

is the solution of the time-dependent parabolic Anderson equation (or stochastic heat equation)

$$
u_{\beta}(t, x)=1+\kappa \int_{0}^{t} \Delta u_{\beta}(s, x) d s+\beta \int_{0}^{t} u_{\beta}(s, x) \partial d W_{x}(s)
$$

The functions $u_{\beta}(t, x)$ and $Z_{\kappa, \beta, t}(x)$ thus have the same distribution so one can make use of the properties of $u(t, x)$ and apply them to $Z_{\kappa, \beta, t}(x)$. In particular, as in the case of the a.s. Lyapunov exponent, the limit

$$
\Psi(\kappa, \beta)=\lim _{T \rightarrow \infty} T^{-1} \ln Z_{\kappa, \beta, T}
$$

exists a.s. Brownian scaling gives that

$$
\Psi(\kappa, \beta)=\beta^{2} \Psi\left(\beta^{-2} \kappa, 1\right)=\beta^{2} \lambda\left(\beta^{-2} \kappa\right) .
$$

Thus, by (96), it follows that

$$
\Psi(\kappa, \beta) \sim \frac{\alpha^{2} \beta^{2}}{4 \ln \left(\beta^{2} / \kappa\right)}, \quad \frac{\beta^{2}}{\kappa} \longrightarrow \infty .
$$


We will use the notation $E_{\kappa}, \mu_{\kappa, \beta, T}$ and $Z_{\kappa, \beta, T}$ when $x=0$.

Similar discrete models have received considerable attention in recent years. Earlier works by [87, 96-98] and many others focused on a discrete model. In this model, the path space is $\mathcal{O}=\left\{\omega=\left(\omega_{n}\right): \omega_{n} \in \mathbf{Z}^{d}, n \geq 1\right\}$ and $S_{n}(\omega)=\omega_{n}$ is the coordinate map. The process

$$
\left(\left\{S_{n}: n \geq 0\right\},\left\{P_{x}: x \in \mathbf{Z}^{d}\right\}\right)
$$

is simple random walk started at $x$ in $\mathbf{Z}^{d}$. The random media $\left\{\xi(x, n): x \in \mathbf{Z}^{d}, n \geq 1\right\}$ consist of iid random variables, defined on a probability space $(\Omega, Q)$. A typical choice would be standard centered Gaussians so that the log moment generating function $\nu(\beta)=\beta^{2} / 2$ defined by

$$
e^{\beta^{2} / 2}=E\left[e^{\beta \xi(x, n)}\right]
$$

is defined for all $\beta \in \mathbf{R}$. Then the discrete polymer measure on $\Omega$ is defined by

$$
\mu_{\beta, n}(d \omega)=Z_{n}(\beta)^{-1} \exp \left\{\sum_{j=1}^{n}\left(\beta \xi\left(S_{j}, j\right)-\frac{\beta^{2}}{2}\right)\right\} P(d \omega),
$$

where the partition function $Z_{n}(\beta)=P\left[e^{\sum_{j=1}^{n}\left(\beta \xi\left(S_{j}, j\right)-\beta^{2} / 2\right)}\right]$. The questions now focus on the behavior of the paths $\left\{S_{j}\right.$ : $1 \leq j \leq n\}$ with respect to the measure $\mu_{\beta, n}$. It was observed by Bolthausen [96] that $Z_{n}(\beta)$ is a positive martingale with respect to the $\sigma$-field

$$
\mathscr{F}_{n}=\sigma\left\{\xi(x, j): x \in \mathbf{Z}^{d}, 1 \leq j \leq n\right\} .
$$

Moreover, for $Z_{\infty}(\beta)=\lim _{n \rightarrow \infty} Z_{n}(\beta)$, the event $\left\{Z_{\infty}(\beta)=\right.$ $0\}$ is measurable with respect to the tail field

$$
\mathscr{T}=\bigcap_{n=1}^{\infty} \sigma\left\{\xi(x, j): x \in \mathbf{Z}^{d}, j \geq n\right\}
$$

This implies $Q\left(Z_{\infty}(\beta)=0\right)=0$ or 1 . The case $Q\left(Z_{\infty}(\beta)=\right.$ $0)=0$ is referred to as weak disorder and $Q\left(Z_{\infty}(\beta)=0\right)=$ 1 is called strong disorder. The behavior of $S_{n}$ under $\mu_{\beta, n}$ is reflected in the behavior of $Z_{n}(\beta)$. For example, denote the product measure of $\mu_{\beta, n}$ with itself by $\mu_{\beta, n}^{\otimes 2}$. With this notation, define the overlap by

$$
I_{n}=\mu_{\beta, n-1}^{\otimes 2}\left(S_{n}=\widetilde{S}_{n}\right) .
$$

Then, a result of Comets-Shiga-Yoshida says that

$$
\left\{Z_{\infty}(\beta)=0\right\}=\left\{\sum_{n=1}^{\infty} I_{n}=\infty\right\}, \quad Q \text {-a.s. }
$$

and in the case of weak disorder there exist positive constants $c_{1}, c_{2}$ such that for all sufficiently large $n$,

$$
-c_{1} \log Z_{n}(\beta) \leq \sum_{j=1}^{n} I_{j} \leq-c_{2} \log Z_{n}(\beta), \quad \text { Q-a.s. }
$$

The outcome $Q\left(\sum_{n=1}^{\infty} I_{n}=\infty\right)$ is called weak localization.
Returning to the continuous model, $Z_{\kappa, \beta, T} e^{-\beta^{2} T / 2}$ is a positive martingale with respect to $\mathscr{F}_{T}=\sigma\left\{W_{x}(s): x \in \mathbf{Z}^{d}, 0 \leq\right.$ $s \leq T\}$ which therefore converges, $\lim _{T \rightarrow \infty} Z_{\kappa, \beta, T} e^{-\beta^{2} T / 2}=$ $Z_{\infty}$. Again the weak disorder-strong disorder distinction is defined by the dichotomy $Q\left(Z_{\infty}=0\right)=0$ or $Q\left(Z_{\infty}=0\right)=1$. Denote the product measure of $\mu_{\kappa, \beta, n}$ with itself by $\mu_{\kappa, \beta, n}^{\otimes 2}$. In [99], two versions of the overlap were considered as

$$
\begin{aligned}
J_{\kappa, \beta, T} & \equiv \frac{1}{T} \int_{0}^{T} \mu_{\kappa, \beta, T}^{\otimes 2}(X(t)=Y(t)) d t, \\
I_{\kappa, \beta, T} & =\frac{1}{T} \int_{0}^{T} \mu_{\kappa, \beta, t}^{\otimes 2}(X(t)=Y(t)) d t .
\end{aligned}
$$

The quantity $J_{\kappa, \beta, T}$ measures, for two independent samples, $X$ and $Y$, sharing the same environment, the proportion of time spent together. The version $I_{\kappa, \beta, T}$ of the overlap measures the amount of time up to $T$ that the endpoints of independent samples drawn with respect to the measure $\mu_{\kappa, \beta, t}$ agree. By taking the logarithmic Malliavin derivative of the partition function with respect to $W_{x}(t)$, one arrives naturally at $J_{\kappa, \beta, T}$. By taking the logarithmic Itô derivative of the partition function, one arrives at $I_{\kappa, \beta, T}$. The overlap occurs in statistical mechanics in a natural way and of course the present model is essentially similar to standard statistical mechanical models in that it involves a Gibbs measure. In statistical mechanics, counterparts of these overlaps can be found, for the Sherrington-Kirkpatrick model and other ones for disordered systems. Coming via integration by parts, the first overlap has been the most successful in the last decade [100-103] to study the low temperature regime.

Now in $[68,84,87]$, it was demonstrated that strong disorder and weak localization are equivalent as

$$
Q\left(Z_{\infty}=0\right)=1 \quad \text { iff } \int_{0}^{\infty} \mu_{\kappa, \beta, t}^{\otimes 2}(X(t)=Y(t)) d t=\infty .
$$

In [85], the stronger result was established as

$$
\begin{aligned}
& Q\left(Z_{\infty}=0\right)=1 \text { implies } \\
& \limsup _{t \rightarrow \infty} \sup _{x \in Z^{d}} \mu_{\kappa, \beta, t}(X(t)=x) \geq c>o,
\end{aligned}
$$

for some $c>0$.

There are several related results on localization. Strong concentration for the directed polymer in a random environment for parabolic Anderson model (space dependent only) with a Pareto potential was established in [17]. The main difference is that there the favourable sites in the environment have a simple characterization in terms of the potential. In the discrete time case with heavy tailed potentials, see [82] for similar conclusions. When the tails are less heavy, the favourite corridors can no longer be characterized by maxima of the potential, and they are no longer explicit, but complete site localization can still be proved [95]. Note that in the discrete case, only little is known on the random geodesics [104] in first passage percolation, which are the zero-temperature 
favourite paths. For the solution of the KPZ equation in one dimension, the distribution of the favourite end-point has been recently computed in [93], and it is the argmax of an Airy $_{2}$ process minus a parabola.

In [99], the behavior of the overlaps $J_{\kappa, \beta, T}$ and $I_{\mathcal{\kappa}, \beta, T}$ was quantified. This used, among other things, the relation (182) and the identity

$$
\frac{\partial}{\partial \beta} E\left[\ln Z_{\kappa, \beta, T}\right]=\beta T\left[1-E\left[J_{\kappa, \beta, T}\right]\right]
$$

which is obtained by the Malliavin calculus and integration by parts. Observe from (194) that

$$
\begin{aligned}
\Psi(\kappa, \beta) & =\lim _{T \rightarrow \infty} T^{-1} E\left[\ln Z_{\kappa, \beta, T}\right] \\
& =\lim _{T \rightarrow \infty} \int_{0}^{\beta} r\left[1-E\left[J_{\kappa, r, T}\right]\right] d r .
\end{aligned}
$$

Theorem 15. (i) For all $\beta$ and $\kappa$ the limit

$$
\widetilde{I}_{\kappa, \beta, \infty}=\lim _{T \rightarrow \infty} I_{\kappa, \beta, T}
$$

exists almost surely, is nonrandom, and is equal to

$$
\widetilde{I}_{\kappa, \beta, \infty}=1-\frac{2}{\beta^{2}} \Psi(\kappa, \beta) \text {. }
$$

(ii) The limit

$$
\widetilde{J}_{\kappa, \beta, \infty}=\lim _{T \rightarrow \infty} E\left[J_{\kappa, \beta, T}\right]
$$

exists for all $\kappa$ and all $\beta$ except for an at most countable set of values of $\beta^{2} / \kappa$, and

$$
\widetilde{J}_{\kappa, \beta, \infty}=1-\beta^{-1} \frac{\partial}{\partial \beta} \Psi(\kappa, \beta) .
$$

(iii) As $\beta^{2} / \kappa \rightarrow \infty$, we have

$$
\widetilde{I}_{\kappa, \beta, \infty}=1-\frac{\alpha^{2}}{2 \ln \left(\beta^{2} / \kappa\right)}(1+o(1)),
$$

and

(iv)

$$
\widetilde{J}_{\kappa, \beta, \infty}=1-\mathcal{O}\left(\frac{1}{\ln \left(\beta^{2} / \kappa\right)}\right) .
$$

(v) Weak versus Strong Disorder. From (196) and (197) it follwos that

$$
\Psi(\kappa, \beta)=\frac{\beta^{2}}{2} \Longleftrightarrow \frac{\beta^{2}}{\kappa} \leq \Upsilon_{c},
$$

for some critical value $\Upsilon_{c} \in[0, \infty)$ depending only on the dimension. This is the weak disorder regime.

In dimension $d \geq 3$, it is known by second moment method [85] that $Z_{\kappa, \beta, T} \exp \left\{-T \beta^{2} / 2\right\}$ converges to a positive limit, so that the equality holds in the left member of (202) for $\beta^{2} / \kappa$ small. Hence, $\Upsilon_{c}>0$ in that case.

There are many interesting open problems on Anderson polymers. (i) The favorite point is defined in [99] as

$$
x^{*}(t)=\operatorname{argmax}\left\{\mu_{\kappa, \beta, t}(X(t)=x): x \in \mathbf{Z}^{d}\right\} .
$$

The proper scaling for $x^{*}(t)$ with respect to $\mu_{\kappa, \beta, t}$ is unknown.

(ii) The favorite path was defined in [99] by

$$
y_{T}^{*}(t)=\operatorname{argmax}\left\{\mu_{\kappa, \beta, T}(X(t)=x): x \in \mathbf{Z}^{d}\right\},
$$

and little is known about the path $Y_{T}^{*}(t), 0 \leq t \leq T$.

(iii) The proper scaling for $X(t)$ with respect to $\mu_{\kappa, \beta, t}$ is also unknown.

(iv) In dimension $d=1,2$, it is expected, in view of results for discrete models [90], as mentioned above, that $\Upsilon_{c}=0$, but this remains open.

\section{Acknowledgment}

M. Cranston was supported by a grant from NSF, DMS 0854940 .

\section{References}

[1] C. Henley and D. Huse, "Pinning and roughening of domain walls in ising systems," Physical Review Letters, vol. 54, no. 5, pp. 2708-2711, 1985.

[2] F. Spitzer, Principles of Random Walk, Springer-Verlag, New York, NY, USA, 1976.

[3] D. A. Dawson and E. A. Perkins, "Long-time behavior and coexistence in a mutually catalytic branching model," The Annals of Probability, vol. 26, no. 3, pp. 1088-1138, 1998.

[4] A.-L. Barabási and H. E. Stanley, Fractal Concepts in Surface Growth, Cambridge University Press, Cambridge, UK, 1995.

[5] R. A. Carmona, S. A. Grishin, and S. A. Molchanov, "Massively parallel simulations of motions in a Gaussian velocity field," in Stochastic Modelling in Physical Oceanography, vol. 39 of Progress in Probability, pp. 47-68, Birkhäuser Boston, Boston, Mass, USA, 1996.

[6] S. Molchanov, "Topics in statistical oceanography," in Stochastic Modelling in Physical Oceanography, vol. 39, pp. 343-380, Birkhäuser, Boston, Mass, USA, 1996.

[7] B. Bailey, Fast Dynamos. Theory of Solar and Planetary Dynamos, NATO ASI, ed M.R.E. Proctor, University of Cambridge Press, Cambridge, UK, 1993.

[8] P. K. S. Dittrikh, S. A. Molchanov, A. A. Ruzmaikin, and D. D. Sokolov, "Stationary distribution of the value of the magnetic field in a random flow," Magnitnaya Gidrodinamika, no. 3, pp. 9-12, 1988.

[9] S. A. Molchanov and A. Ruzmaikin, "Lyapunov exponents and distributions of magnetic fields in dynamo models," in The Dynkin Festschrift, vol. 34 of Progress in Probability, pp. 287-306, Birkhäuser, Boston, Mass, USA, 1994.

[10] S. A. Molchanov, A. Ruzmaikin, and D. Sokolov, "Shortcorrelated random flow as a fast dynamo," Soviet PhysicsDoklady, vol. 103, pp. 121-126, 1986.

[11] S. A. Molchanov, A. Ruzmaikin, D. Sokolov, and Y. Zeldovich, Intermittency, Diffusion and Generation in Non-Stationary Random Medium, vol. 7 of Mathematical Physics Review, Harwood Academic, New York, NY, USA, 1988. 
[12] M. Biskup and W. König, "Long-time tails in the parabolic Anderson model with bounded potential," The Annals of Probability, vol. 29, no. 2, pp. 636-682, 2001.

[13] J. Gärtner, W. König, and S. Molchanov, "Geometric characterization of intermittency in the parabolic Anderson model," The Annals of Probability, vol. 35, no. 2, pp. 439-499, 2007.

[14] J. Gärtner and S. A. Molchanov, "Parabolic problems for the Anderson model. I. Intermittency and related topics," Communications in Mathematical Physics, vol. 132, no. 3, pp. 613-655, 1990.

[15] R. van der Hofstad, W. König, and P. Mörters, “The universality classes in the parabolic Anderson model," Communications in Mathematical Physics, vol. 267, no. 2, pp. 307-353, 2006.

[16] W. König, "The parabolic Anderson model and its universality classes," in Probability and Mathematical Physics, vol. 42 of CRM Proceedings and Lecture Notes, pp. 283-298, American Mathematical Society, Providence, RI, USA, 2007.

[17] W. König, H. Lacoin, P. Mörters, and N. Sidorova, "A two cities theorem for the parabolic Anderson model," The Annals of Probability, vol. 37, no. 1, pp. 347-392, 2009.

[18] N. Ikeda and S. Watanabe, Stochastic Differential Equations and Diffusion Processes, North Holland, 1981.

[19] R. A. Carmona and S. A. Molchanov, "Parabolic Anderson problem and intermittency," Memoirs of the American Mathematical Society, vol. 108, no. 518, p. 125, 1994.

[20] A. M. Etheridge and K. Fleischmann, "Compact interface property for symbiotic branching," Stochastic Processes and their Applications, vol. 114, no. 1, pp. 127-160, 2004.

[21] F. Aurzada and L. Döring, "Intermittency and ageing for the symbiotic branching model," Annales de l'Institut Henri Poincaré, vol. 47, no. 2, pp. 376-394, 2011.

[22] J. Blath, L. Döring, and A. Etheridge, "On the moments and the interface of the symbiotic branching model," The Annals of Probability, vol. 39, no. 1, pp. 252-290, 2011.

[23] J. Blath and M. Ortgiese, "Properties of the interface of the symbiotic branching model," preprint.

[24] D. A. Dawson, K. Fleischmann, L. Mytnik, E. A. Perkins, and J. Xiong, "Mutually catalytic branching in the plane: uniqueness," Annales de l'Institut Henri Poincaré. Probabilités et Statistiques, vol. 39, no. 1, pp. 135-191, 2003.

[25] L. Mytnik, "Uniqueness for a mutually catalytic branching model," Probability Theory and Related Fields, vol. 112, no. 2, pp. 245-253, 1998.

[26] T. Shiga, "Stepping stone models in population genetics and population dynamics," in Stochastic Processes in Physics and Engineering (Bielefeld, 1986), vol. 42 of Applications of Mathematics, pp. 345-355, Reidel, Dordrecht, The Netherlands, 1988.

[27] J. Gärtner and F. den Hollander, "Intermittency in a catalytic random medium," The Annals of Probability, vol. 34, no. 6, pp. 2219-2287, 2006.

[28] J. Gartner, F. den Hollander, and G. Maillard, "Quenched Lyapunov exponent for the parabolic Anderson model in a dynamic random environment," Probability in Complex Physical Systems, vol. 11, pp. 159-193, 2012.

[29] J. Gärtner, F. den Hollander, and G. Maillard, "Intermittency of catalysts: voter model," The Annals of Probability, vol. 38, no. 5, pp. 2066-2102, 2010.

[30] J. Gartner, F. den Hollander, and G. Maillard, "Intermittency on catalysts: threedimensional symmetric exclusion," Electronic Journal of Probability, vol. 14, no. 72, pp. 2091-2129, 2009.
[31] J. Gärtner, F. den Hollander, and G. Maillard, "Intermittency on catalysts: symmetric exclusion," Electronic Journal of Probability, vol. 12, no. 18, pp. 516-573, 2007.

[32] A. Greven and F. den Hollander, "Phase transitions for the long-time behavior of interacting diffusions," The Annals of Probability, vol. 35, no. 4, pp. 1250-1306, 2007.

[33] M. Birkner, "A condition for weak disorder for directed polymers in random environment," Electronic Communications in Probability, vol. 9, pp. 22-25, 2004.

[34] M. Cranston and S. A. Molchanov, "On phase transitions and limit theorems for homopolymers," in Probability and Mathematical Physics, vol. 42 of CRM Proceedings and Lecture Notes, pp. 97-112, American Mathematical Society, Providence, RI, USA, 2007.

[35] J. D. Esary, F. Proschan, and D. W. Walkup, "Association of random variables, with applications," Annals of Mathematical Statistics, vol. 38, pp. 1466-1474, 1967.

[36] C. M. Newman, "Normal fluctuations and the FKG inequalities," Communications in Mathematical Physics, vol. 74, no. 2, pp. 119-128, 1980.

[37] L. D. Pitt, "Positively correlated normal variables are associated," The Annals of Probability, vol. 10, no. 2, pp. 496-499, 1982.

[38] T. M. Liggett, "An improved subadditive ergodic theorem," The Annals of Probability, vol. 13, no. 4, pp. 1279-1285, 1985.

[39] M. Cranston, T. S. Mountford, and T. Shiga, "Lyapunov exponents for the parabolic Anderson model," Acta Mathematica Universitatis Comenianae, vol. 71, no. 2, pp. 163-188, 2002.

[40] C. Bezuidenhout and G. Grimmett, "The critical contact process dies out," The Annals of Probability, vol. 18, no. 4, pp. 1462-1482, 1990.

[41] R. Durrett, "Oriented percolation in two dimensions," The Annals of Probability, vol. 12, no. 4, pp. 999-1040, 1984.

[42] R. .A Carmona, L. Koralov, and S. Molchanov, "Asymptotics for the almost sure Lyapunov exponent for the solution of the parabolic Anderson problem," Random Operators and Stochastic Equations, vol. 9, no. 1, pp. 77-86, 2001.

[43] R. J. Adler, An Introduction to Continuity, Extrema, and Related Topics for General Gaussian Processes, vol. 12 of Lecture Notes Monograph Series, Institute of Mathematical Statistics, Hayward, Calif, USA, 1990.

[44] K. Johansson, "Transversal fluctuations for increasing subsequences on the plane," Probability Theory and Related Fields, vol. 116, no. 4, pp. 445-456, 2000.

[45] C. Licea, C. M. Newman, and M. S. T. Piza, "Superdiffusivity in first-passage percolation," Probability Theory and Related Fields, vol. 106, no. 4, pp. 559-591, 1996.

[46] T. Furuoya and T. Shiga, "Sample Lyapunov exponent for a class of linear Markovian systems over $Z^{d}$," Osaka Journal of Mathematics, vol. 35, no. 1, pp. 35-72, 1998.

[47] T. Shiga, "Ergodic theorems and exponential decay of sample paths for certain interacting diffusion systems," Osaka Journal of Mathematics, vol. 29, no. 4, pp. 789-807, 1992.

[48] T. Shiga and A. Shimizu, "Infinite-dimensional stochastic differential equations and their applications," Journal of Mathematics of Kyoto University, vol. 20, no. 3, pp. 395-416, 1980.

[49] J. T. Cox and A. Greven, "Ergodic theorems for infinite systems of locally interacting diffusions," The Annals of Probability, vol. 22, no. 2, pp. 833-853, 1994.

[50] T. M. Liggett, Interacting Particle Systems, Classics in Mathematics, Springer, Berlin, Germany, 2005. 
[51] T. M. Liggett, Stochastic Interacting Systems: Contact, Voter and Exclusion Processes. Grundlehren der Mathematischen Wissenschaften, vol. 324 of Funda Principles of Mathematical Sciences, Springer, Berlin, Germany, 1999.

[52] M. Cranston and S. Molchanov, "Limit laws for sums of products of exponentials of iid random variables," Israel Journal of Mathematics, vol. 148, pp. 115-136, 2005.

[53] G. Ben Arous, L. V. Bogachev, and S. A. Molchanov, "Limit theorems for sums of random exponentials," Probability Theory and Related Fields, vol. 132, no. 4, pp. 579-612, 2005.

[54] G. Ben Arous, S. Molchanov, and A. F. Ramírez, "Transition from the annealed to the quenched asymptotics for a random walk on random obstacles," The Annals of Probability, vol. 33, no. 6, pp. 2149-2187, 2005.

[55] M. Cranston and S. Molchanov, "Quenched to annealed transition in the parabolic Anderson problem," Probability Theory and Related Fields, vol. 138, no. 1-2, pp. 177-193, 2007.

[56] G. Grimmett and H. Kesten, "First-passage percolation, network flows and electrical resistances," Zeitschrift für Wahrscheinlichkeitstheorie und Verwandte Gebiete, vol. 66, no. 3, pp. 335-366, 1984.

[57] J. T. Cox and G. Grimmett, "Central limit theorems for associated random variables and the percolation model," The Annals of Probability, vol. 12, no. 2, pp. 514-528, 1984.

[58] R. M. Balan, "A strong invariance principle for associated random fields," The Annals of Probability, vol. 33, no. 2, pp. 823840, 2005.

[59] T.-S. Kim, "The invariance principle for associated random fields," The Rocky Mountain Journal of Mathematics, vol. 26, no. 4, pp. 1443-1454, 1996.

[60] C. M. Newman and A. L. Wright, "An invariance principle for certain dependent sequences," The Annals of Probability, vol. 9, no. 4, pp. 671-675, 1981.

[61] A. R. Dabrowski and A. Jakubowski, "Stable limits for associated random variables," The Annals of Probability, vol. 22, no. 1, pp. 1-16, 1994.

[62] P. Lèvy, Problèmes Complets D'analyse Fonctionelle, GauthiersVillars, 1951.

[63] M. Talagrand, "Concentration of measure and isoperimetric inequalities in product spaces," Institut des Hautes Études Scientifiques, no. 81, pp. 73-205, 1995.

[64] M. Talagrand, "New concentration inequalities in product spaces," Inventiones Mathematicae, vol. 126, no. 3, pp. 505-563, 1996.

[65] M. Talagrand, "A new look at independence," The Annals of Probability, vol. 24, no. 1, pp. 1-34, 1996.

[66] M. Ledoux, The Concentration of Measure Phenomenon, vol. 89 of Mathematical Surveys and Monographs, American Mathematical Society, 2001.

[67] A. Toubol, "High temperature regime for a multidimensional Sherrington-Kirkpatrick model of spin glass," Probability Theory and Related Fields, vol. 110, no. 4, pp. 497-534, 1998.

[68] C. Rovira and S. Tindel, "On the Brownian-directed polymer in a Gaussian random environment," Journal of Functional Analysis, vol. 222, no. 1, pp. 178-201, 2005.

[69] D. Nualart, The Malliavin Calculus and Related Topics, Springer, Berlin, Germany, 2nd edition, 2006.

[70] A. S. Üstünel and M. Zakai, Transformation of Measure on Wiener Space, Springer, Berlin, Germany, 2000.
[71] I. Ben-Ari, "Large deviations for partition functions of directed polymers in an IID field," Annales de l'Institut Henri Poincaré Probabilités et Statistiques, vol. 45, no. 3, pp. 770-792, 2009.

[72] M. Cranston, D. Gauthier, and T. S. Mountford, "On large deviation regimes for random media models," The Annals of Applied Probability, vol. 19, no. 2, pp. 826-862, 2009.

[73] M. Cranston, D. Gauthier, and T. S. Mountford, "On large deviations for the parabolic Anderson model," Probability Theory and Related Fields, vol. 147, no. 1-2, pp. 349-378, 2010.

[74] Y. Chow and Y. Zhang, "Large deviations in first-passage percolation," The Annals of Applied Probability, vol. 13, no. 4, pp. 1601-1614, 2003.

[75] J.-D. Deuschel and O. Zeitouni, "On increasing subsequences of I.I.D. Samples," Combinatorics, Probability and Computing, vol. 8, no. 3, pp. 247-263, 1999.

[76] G. Grimmett, Percolation, Springer, New York, NY, USA, 1999.

[77] J. M. Hammersley and D. J. A. Welsh, "First-passage percolation, subadditive processes, stochastic networks and generalized renewal theory," in Bernoulli, Bayes, J. Neyman and L. Le Cam, Eds., Laplace Anniversary Volume, Springer, Berlin, Germany, 1965.

[78] R. A. Carmona and F. G. Viens, "Almost-sure exponential behavior of a stochastic Anderson model with continuous space parameter," Stochastics and Stochastics Reports, vol. 62, no. 3-4, pp. 251-273, 1998.

[79] M. Cranston and T. S. Mountford, "Lyapunov exponent for the parabolic Anderson model in $R^{d}$," Journal of Functional Analysis, vol. 236, no. 1, pp. 78-119, 2006.

[80] M. Rael, "Asymptotics of the Lyapunov exponent for the continuum parabolic Anderson model," preprint.

[81] G. Amir, I. Corwin, and J. Quastel, "Probability distribution of the free energy of the continuum directed random polymer in $1+1$ dimensions," Communications on Pure and Applied Mathematics, vol. 64, no. 4, pp. 466-537, 2011.

[82] A. Auffinger and O. Louidor, "Directed polymers in random environment with heavy tails," Communications on Pure and Applied Mathematics. In press.

[83] A. Cadel, S. Tindel, and F. Viens, "Sharp asymptotics for the partition function of some continuous-time directed polymers," Potential Analysis, vol. 29, no. 2, pp. 139-166, 2008.

[84] P. Carmona and Y. Hu, "On the partition function of a directed polymer in a Gaussian random environment," Probability Theory and Related Fields, vol. 124, no. 3, pp. 431-457, 2002.

[85] P. Carmona and Y. Hu, "Strong disorder implies strong localization for directed polymers in a random environment," Latin American Journal of Probability and Mathematical Statistics, vol. 2, pp. 217-229, 2006.

[86] F. Comets, T. Shiga, and N. Yoshida, "Directed polymers in a random environment: path localization and strong disorder," Bernoulli, vol. 9, no. 4, pp. 705-723, 2003.

[87] F. Comets, T. Shiga, and N. Yoshida, "Probabilistic analysis of directed polymers in a random environment: a review," in Stochastic Analysis on Large Scale Interacting Systems, vol. 39 of Advanced Studies in Pure Mathematics., pp. 115-142, Mathematics Society, Tokyo, Japan, 2004.

[88] F. Comets and N. Yoshida, "Directed polymers in random environment are diffusive at weak disorder," The Annals of Probability, vol. 34, no. 5, pp. 1746-1770, 2006.

[89] G. Giacomin, Random Polymer Models, Imperial College Press, 2007. 
[90] H. Lacoin, "New bounds for the free energy of directed polymers in dimension $1+1$ and $1+2$," Communications in Mathematical Physics, vol. 294, no. 2, pp. 471-503, 2010.

[91] D. Marquez-Carreras, R. Rovira, and S. Tindel, "A model of continuous time polymer on the lattice," In press, http:// arxiv.org/abs/0802.3296.

[92] G. Moreno Flores, "Asymmetric directed polymers in random environments," In press, http://arxiv.org/abs/1009.5576.

[93] G. Moreno Flores, J. Quastel, and D. Remenik, "Endpoint distribution of directed polymers in 1+1 dimensions," Preprint, http://arxiv.org/abs/1106.2716.

[94] J. Moriarty and N. O'Connell, "On the free energy of a directed polymer in a Brownian environment," Markov Processes and Related Fields, vol. 13, no. 2, pp. 251-266, 2007.

[95] V. Vargas, "Strong localization and macroscopic atoms for directed polymers," Probability Theory and Related Fields, vol. 138, no. 3-4, pp. 391-410, 2007.

[96] E. Bolthausen, "A note on the diffusion of directed polymers in a random environment," Communications in Mathematical Physics, vol. 123, no. 4, pp. 529-534, 1989.

[97] J. Z. Imbrie and T. Spencer, "Diffusion of directed polymers in a random environment," Journal of Statistical Physics, vol. 52, no. 3-4, pp. 609-626, 1988.

[98] R. Song and X. Y. Zhou, "A remark on diffusion of directed polymers in random environments," Journal of Statistical Physics, vol. 85, no. 1-2, pp. 277-289, 1996.

[99] F. Comets and M. Cranston, "Overlaps and pathwise localization in the anderson polymer model," http://arxiv.org/ abs/1107.2011.

[100] F. Guerra, "Sum rules for the free energy in the mean field spin glass model," in Mathematical Physics in Mathematics and Physics (Siena, 2000), vol. 30 of Fields Institute Communications, pp. 161-170, American Mathematical Society, Providence, RI, USA, 2001.

[101] F. Guerra and F. L. Toninelli, "The thermodynamic limit in mean field spin glass models," Communications in Mathematical Physics, vol. 230, no. 1, pp. 71-79, 2002.

[102] M. Talagrand, Spin Glasses: A Challenge for Mathematicians. Cavity and Mean Field Models, Springer, Berlin, Germany, 2003.

[103] M. Talagrand, “The Parisi formula," Annals of Mathematics, vol. 163, no. 1, pp. 221-263, 2006.

[104] C. Newman, Topics in Disordered Systems. Lectures in Mathematics ETH Zurich, Birkhäuser, Basel, Switzerland, 1997. 


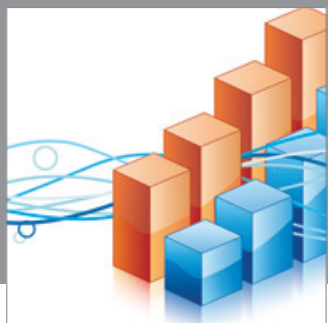

Advances in

Operations Research

mansans

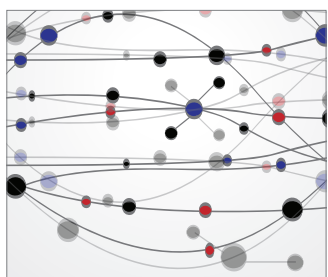

The Scientific World Journal
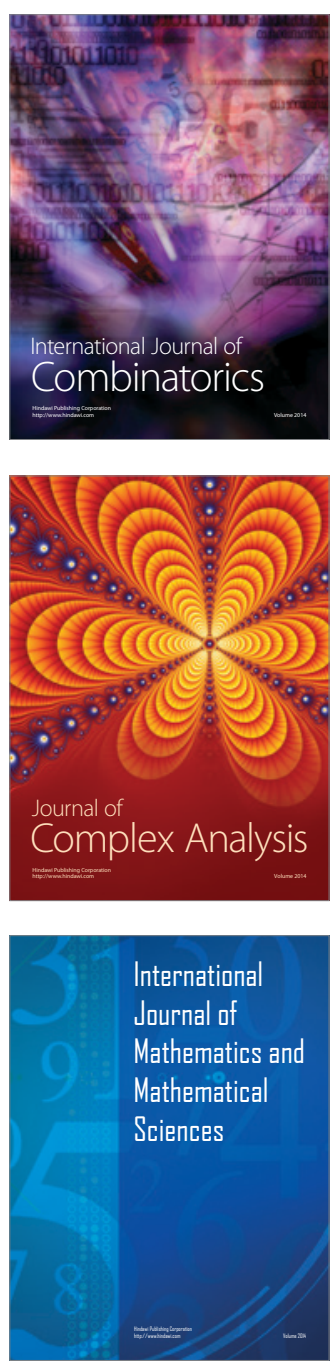
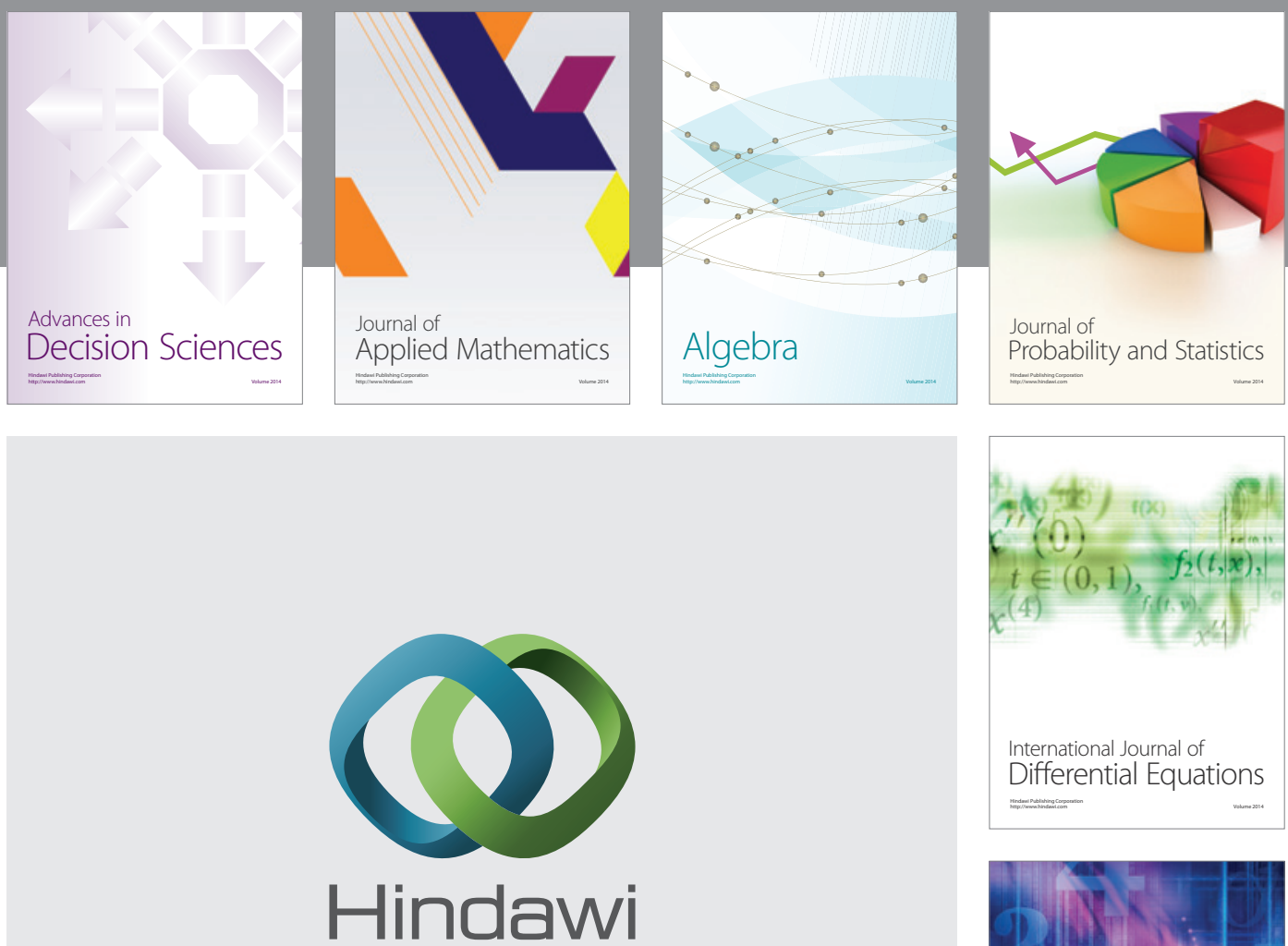

Submit your manuscripts at http://www.hindawi.com
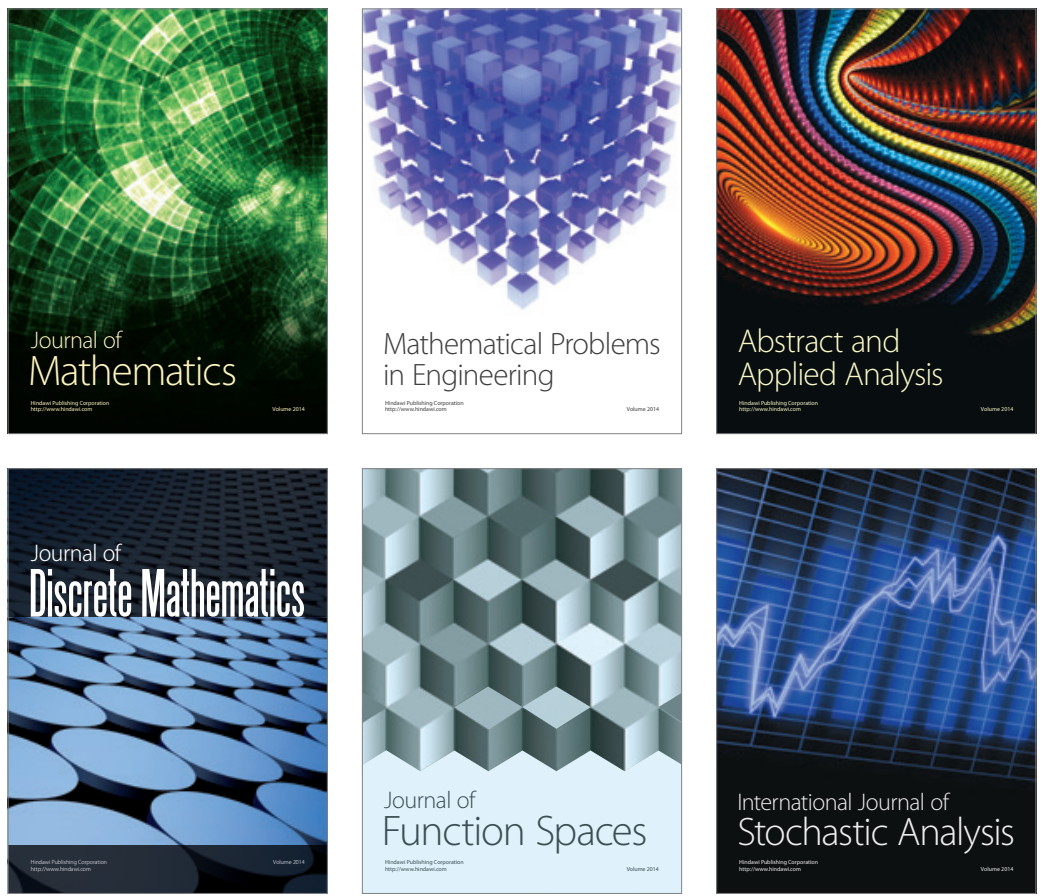

Journal of

Function Spaces

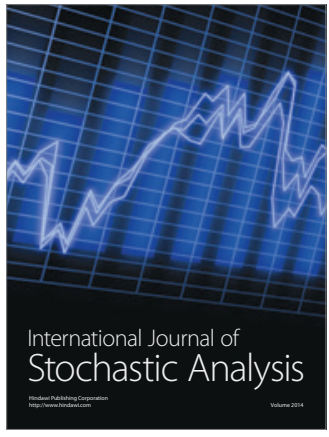

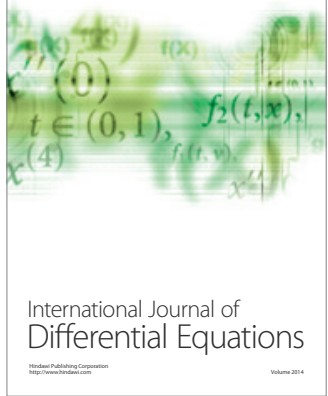
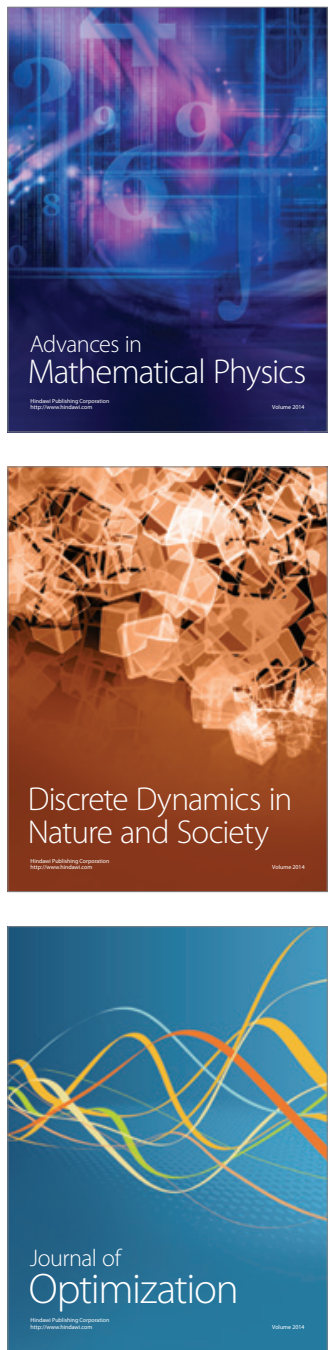